\title{
THE PROSPECTS OF DEVELOPING THE DISTANCE EMPLOYMENT UNDER THE CONDITIONS OF CORONOMICS IN GEORGIA
}

\author{
MURMAN TSARTSIDZE \\ Doctor of Economic Sciences, \\ Associate professor, \\ Ivane Javakhishvili Tbilisi State University, \\ Academician of the Academy of Economic Sciences of Georgia, Georgia \\ murman.tsartsidze@tsu.ge
}

Abstract. In the era of current globalization, when the whole world is faced with the qualitatively new economic crisis, for the purpose of raising the living standards of population the great importance is attached to ensuring their effective employment. The latter belongs to the major social-economic issues and poses quite a serious challenge to a country. The aforesaid is also conditioned by the fact that at the current stage of formation and operation of labour market the unemployment is regarded as the key factor causing poverty. Under the conditions of coronomics the problem is more exacerbated by thousands of workplaces having been temporarily suspended and lost due to the spread of Covid19 within the country.

In such a situation it would be difficult to cope with the unemployment and make progress in effective employment without developing the socio-labour relations and applying its qualitatively new forms. Occasioned by the above mentioned, the paper aims at the profound study of peculiarities of the forms of distance employment, which are widespread in the international practice, and focuses on the formulation of certain directions and recommendations relating to the potential and prospects of their utilization in the country. In particular, on the basis of the research and analysis conducted the following key recommendations and conclusions have been reached:

1. In the contemporary world the expansion of non-standard employment including the distance employment has radically changed the tendencies of developing the labour market. The mentioned forms of employment are characterized by: labour flexibility; the application of non-standard organizational-legal forms of labour; the adaptation of employers and employees to the constantly changing economic conditions; the realization of their interests, etc.;

2. In the developed countries the distance employment greatly contributes to the increase in the overall level and scales of employment. Its main distinctive mark is the existence of workplaces out of enterprise (outworks). Accordingly, the distance employment is examined in the context of non-standard employment represented as the form of long-term labour relations with a single employer, which diverges from the standard model of full, regulated and open employment according to one or several criteria (performance, labour regime, labour hours, working conditions, work outcomes, working hours, work place and its location, social protection, the correlation between the subjects of labour relations, etc.);

3. Based on its attractiveness as well as by popular demand, the distance employment is recently developing and enhancing at a fast pace all over the world. For instance, while at the outset of the 2000s approximately 30.0 million people were employed on distance jobs in the USA and EU countries, in the second decade of XXI century their number increased to $55.0-60.0$ million. The quantity of distance employers is annually growing by $20 \%-30 \%$ worldwide. In 2019 , the number of outworking employees equaled to $39.0 \%$ all over the world. In addition, $28 \%$ more of employees had the right to distance jobs. The USA, Canada, Finland, Denmark and Sweden lead this sphere;

4. The advantages of distance employment are as follows: 1 . On behalf of employees: flexible working hours; the standard, comfortable conditions of employment; the opportunity to get education and participate in the socially useful activities; extra income; contracts with outer organizations; the possibility to schedule the working time and the labour optimization; the provision of high efficiency and quality of work; the maintenance of the personal rhythm, resting and working time and their protection; saving of expenses; the decrease in time of communications; comfortable working space; the lack of corporate culture; the distance employment is the basis of creative self-realization, pleasure and satisfaction for a number of employers. 2.On behalf of employers: employing the professionals and providing the flexibility of personnel; raising the labour productivity and efficiency; ensuring the personnel loyalty; providing the clients with round -the-clock information services; forming the flexible organizational structure for enterprises; work site arrangement, saving rental costs. 3. On behalf 
of a state: the increase in the level of employment and the decrease in the level of unemployment; the development of distance employment involving the disabled persons; the decrease in traffic in the country; the increase in the number of economically active population; scaling down the migration;

5. Apart from the positive points, the expansion of distance employment is related to the significant risk factors, which necessitate the introduction of their controlling mechanism by the state. It should be focused on reducing the aforesaid riskfactors to minimum level and protecting social and legal rights of employees;

6. Occasioned by the goals of ensuring the effective employment as well as according to National Strategy for Labour Market and Employment Policy of Georgia 2019-2023, the provision of developing the non-standard forms of employment assumes the great importance in the country. It is also essential to regularly conduct the impact assessment defining the scales of distance jobs and the pace of their expansion at the level of total employment;

7. In order to conduct the comprehensive analysis and evaluation of distance employment at the overall level of employment, we deem it expedient to make its classification not in terms of the scope of fields but according to the professions, which are currently singled out as being in great demand. Currently, at the labour market of Georgia the following specialties are most demanded by employers: 1 . A specialist in marketing; 2 . Sales manager; 3 . The specialist in the area of internet-technologies; 4. A web-designer; 5. A programmer; 6. Distributer; 7. Tourism ( where the demands have, accordingly, increased on the specialties such as a waiter, a barman, a hotel manager, a guide); 8. Audit; 9. Accountant - financier; 10. A doctor; 11. A lawyer; 12. A teacher; 13. A construction manager; 14. Translator, etc. Exactly the mentioned professions are attractive in terms of developing the distance employment in a number of developed countries abroad;

8. With the aim of raising the efficiency of state programs for employment and regulating the distance employment, the regular researches should be necessarily implemented in the direction of determining the real demands on working forces at the labour market. This is the only way offering the real prospects for developing the distance employment and their involvement in the state and regional programs of employment. The latter will considerably contribute to improving the quality of working life of population and ultimately, the raise of living standards.

KEYWORDS: DISTANCE EMPLOYMENT, NON-STANDARD EMPLOYMENT, LABOUR MARKET, UNEMPLOYMENT, CORONOMICS.

For citation: Tsartsidze, M., (2020). The prospects of developing the distance employment under the conditions of coronomics in Georgia. Globalization and Business, 10. 138-149. (In Georgian) https://doi.org/10.35945/gb.2020.10.017 


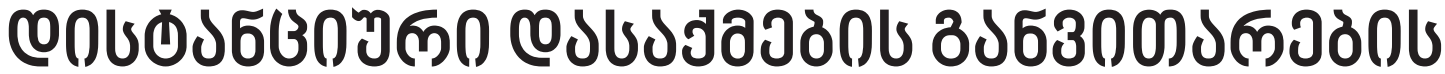

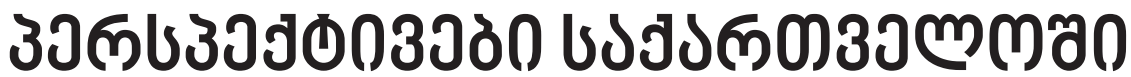

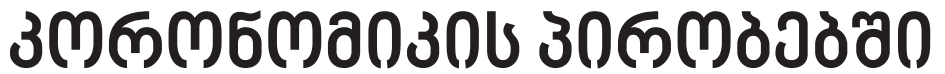

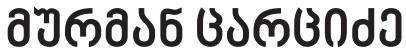

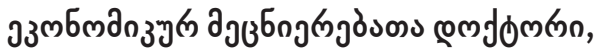

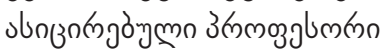

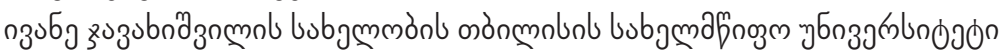

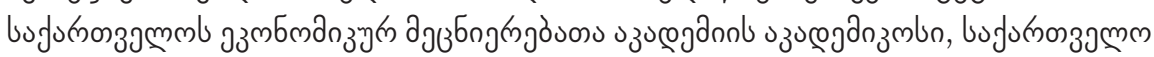 \\ murman.tsartsidze@tsu.ge
}

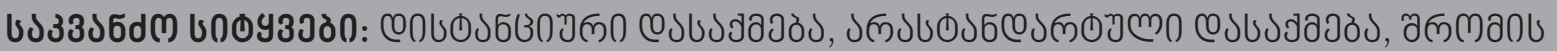

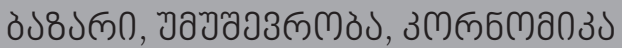

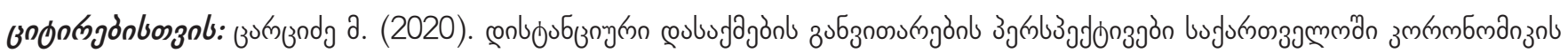

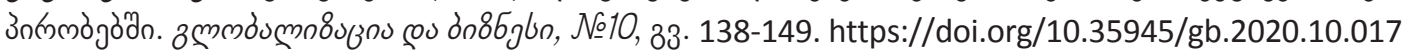

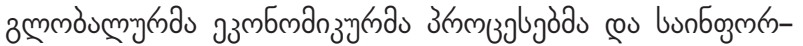

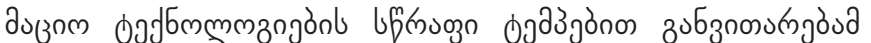

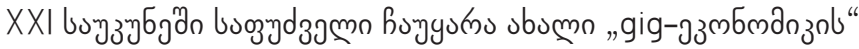

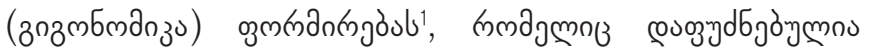

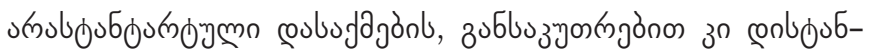

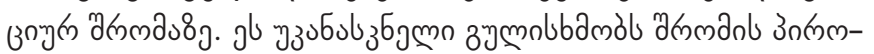

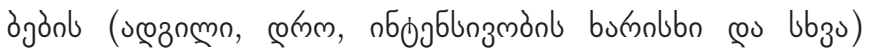

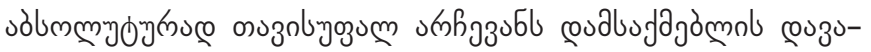

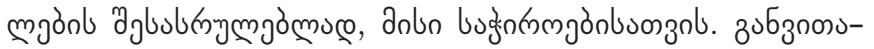

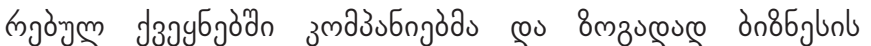

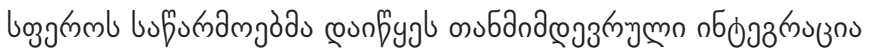

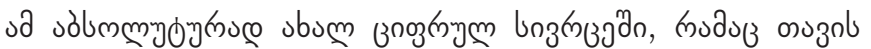

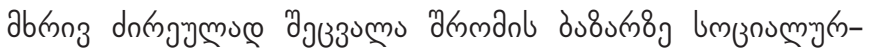

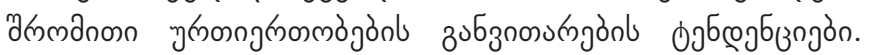

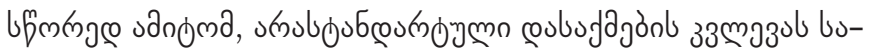

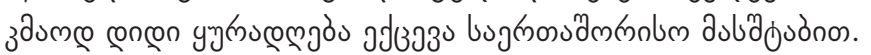

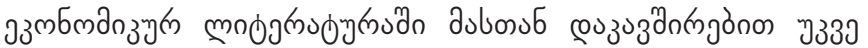

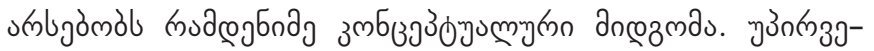

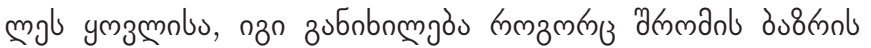

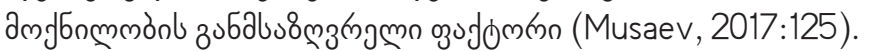

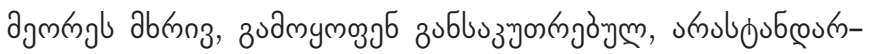

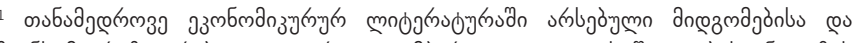

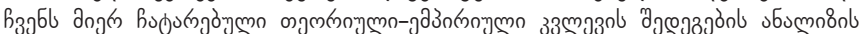

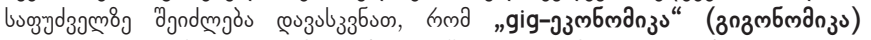

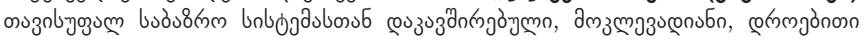

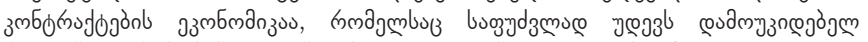

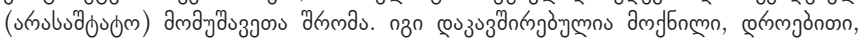

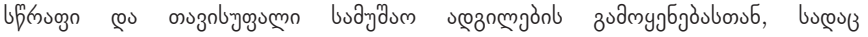

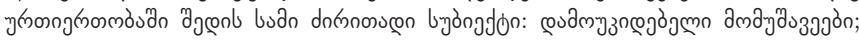

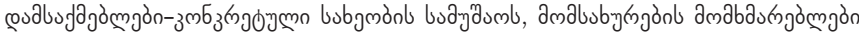

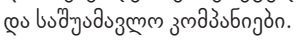

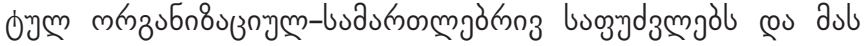

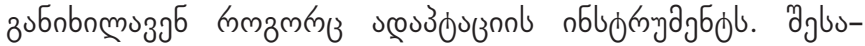

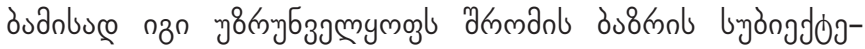
on

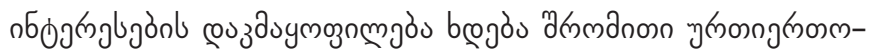

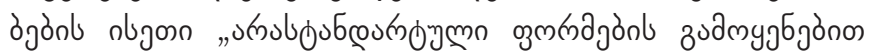

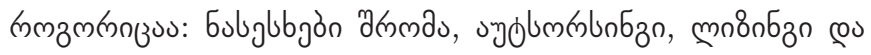

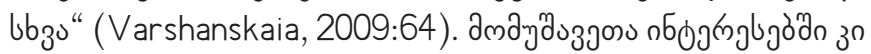

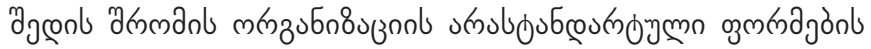

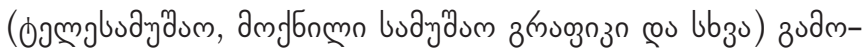

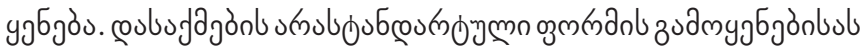

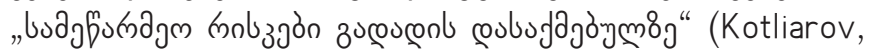

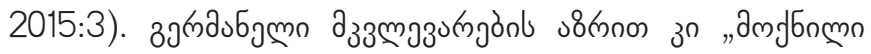

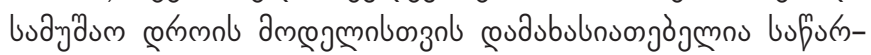
aman coubufajäymol zublbbzuzadymn conmnon ymozbu cennl,

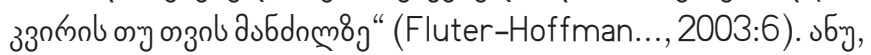

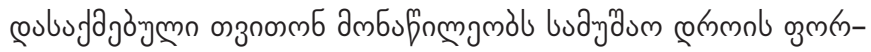

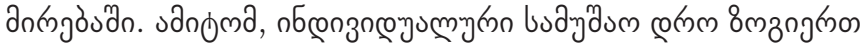

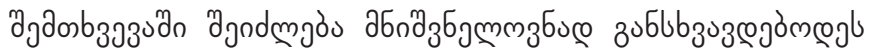

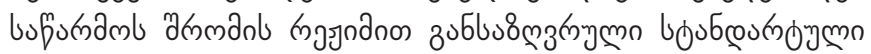

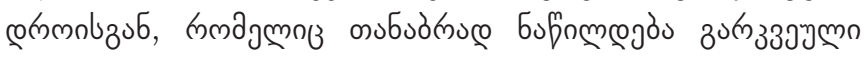

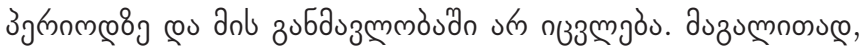

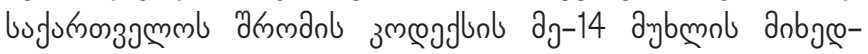

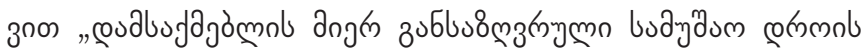

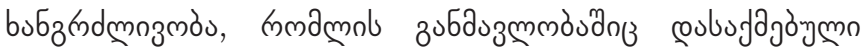

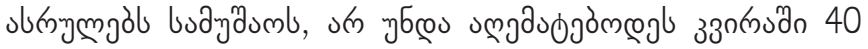

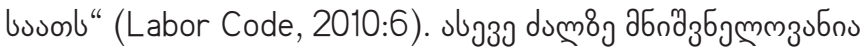

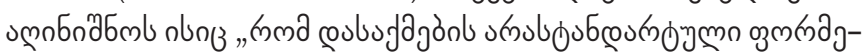

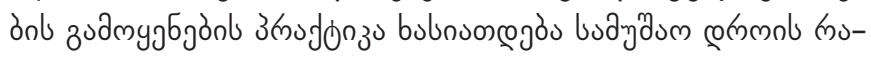




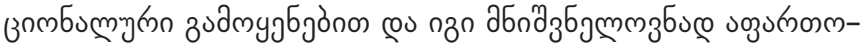

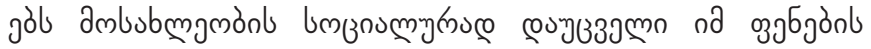

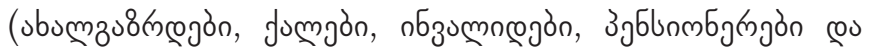

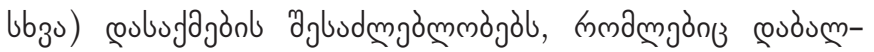

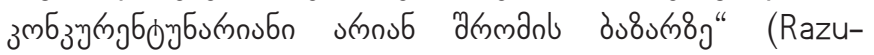

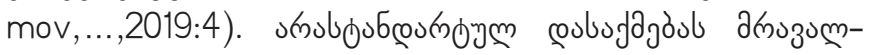

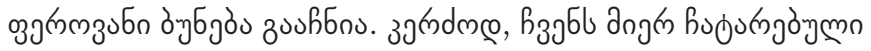

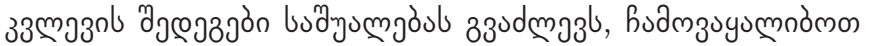

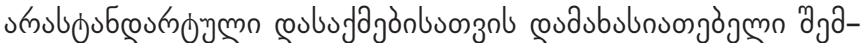

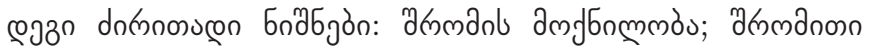

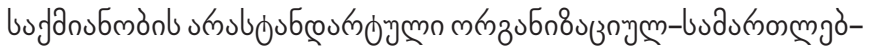

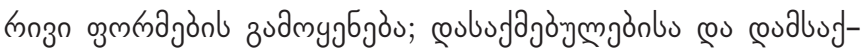

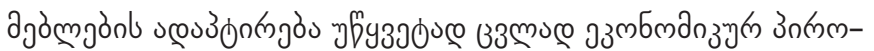

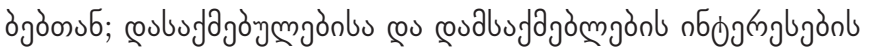

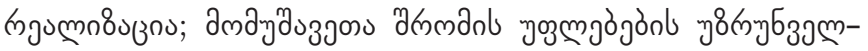

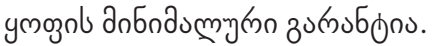

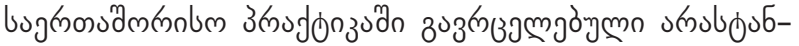

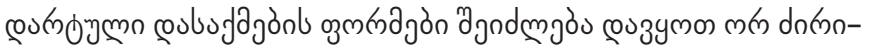

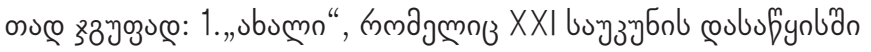

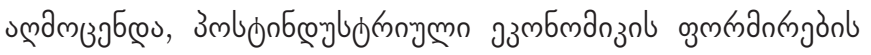

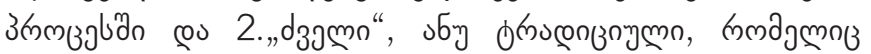

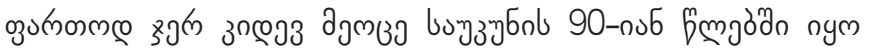

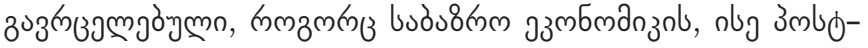

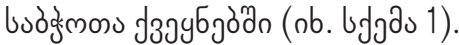

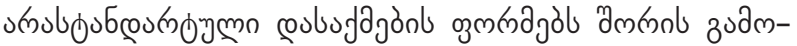

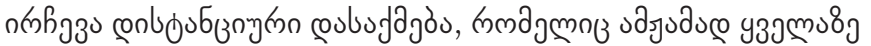

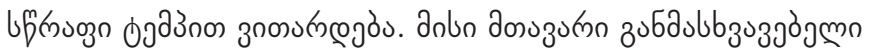

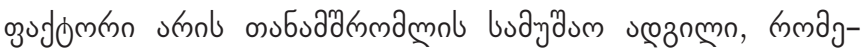

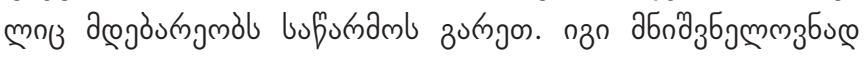

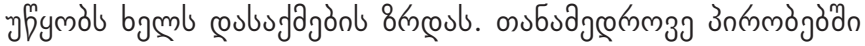

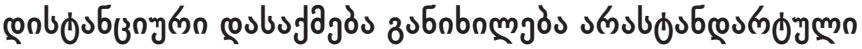

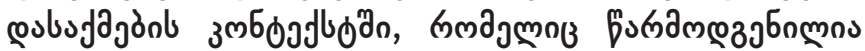

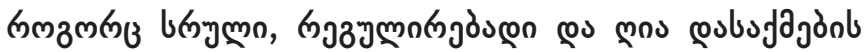

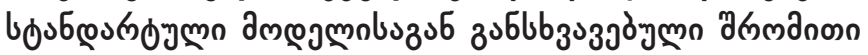

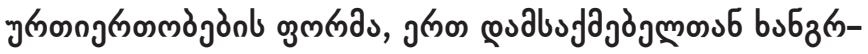

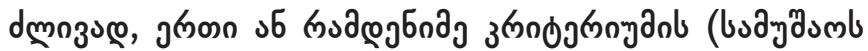

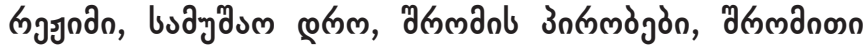

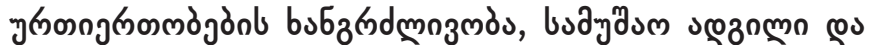

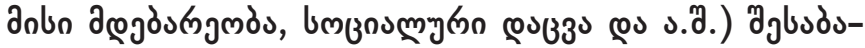
anbus (ob. bjgas 2) (Razumov, ..., 2019:37-57).

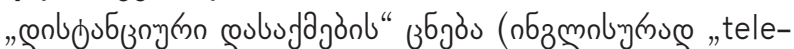

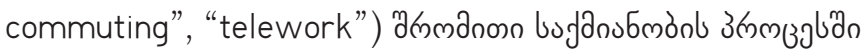

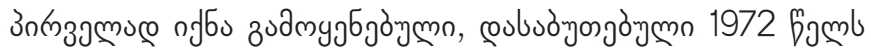

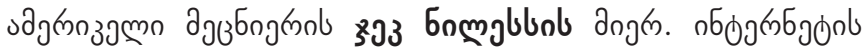

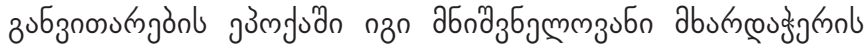

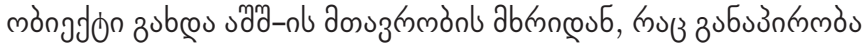

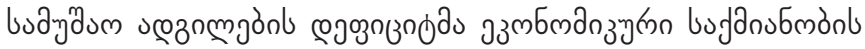

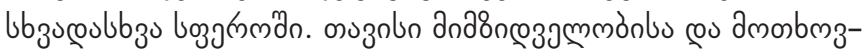

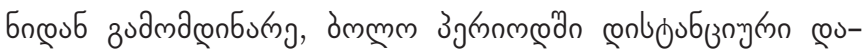

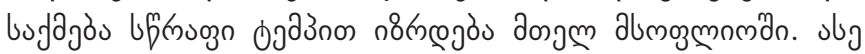

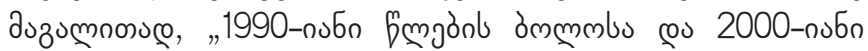

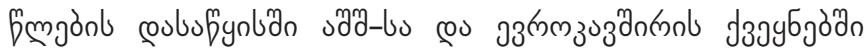

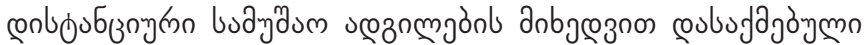

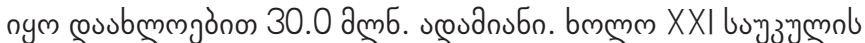

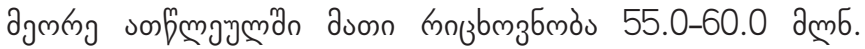

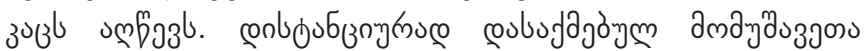

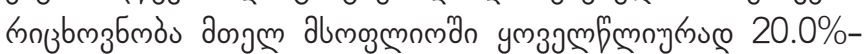

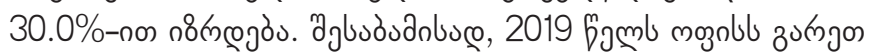

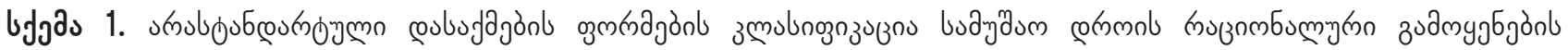
mзumbugmolnom

\begin{tabular}{|c|c|}
\hline \multicolumn{2}{|c|}{ 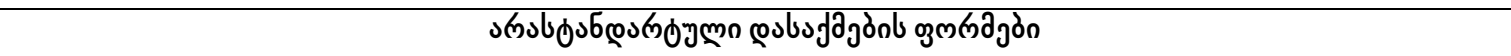 } \\
\hline 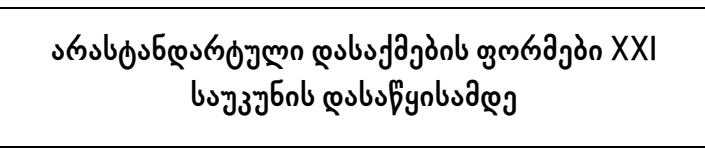 & 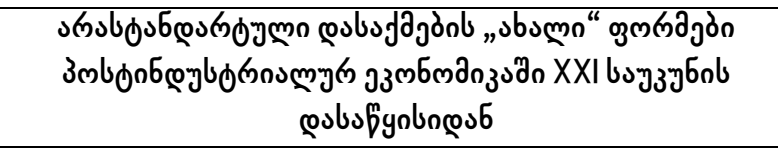 \\
\hline 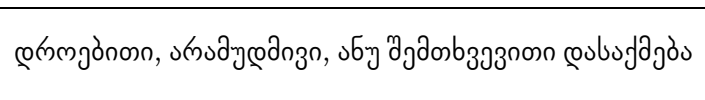 & 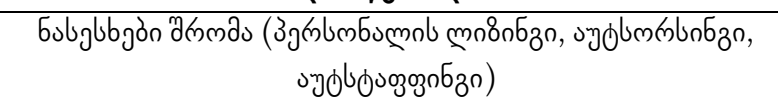 \\
\hline 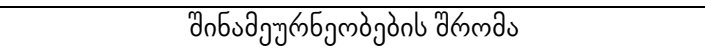 & 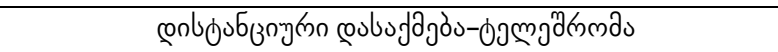 \\
\hline 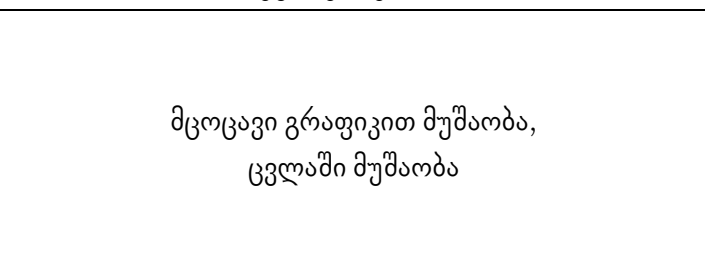 & 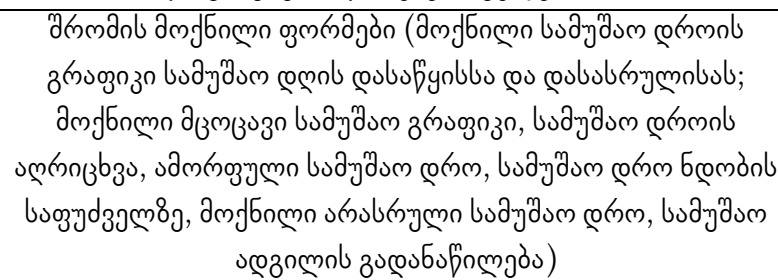 \\
\hline smulumymn coubufajò & gुmnmubligmgon \\
\hline の3noscolotagós & 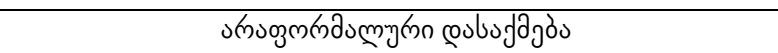 \\
\hline 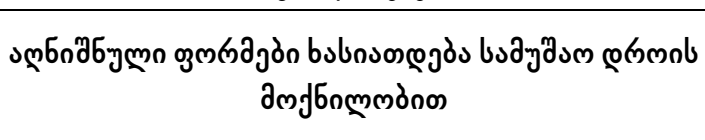 & 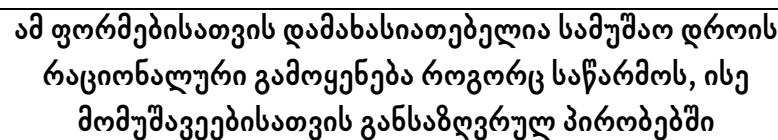 \\
\hline
\end{tabular}




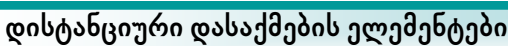

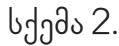

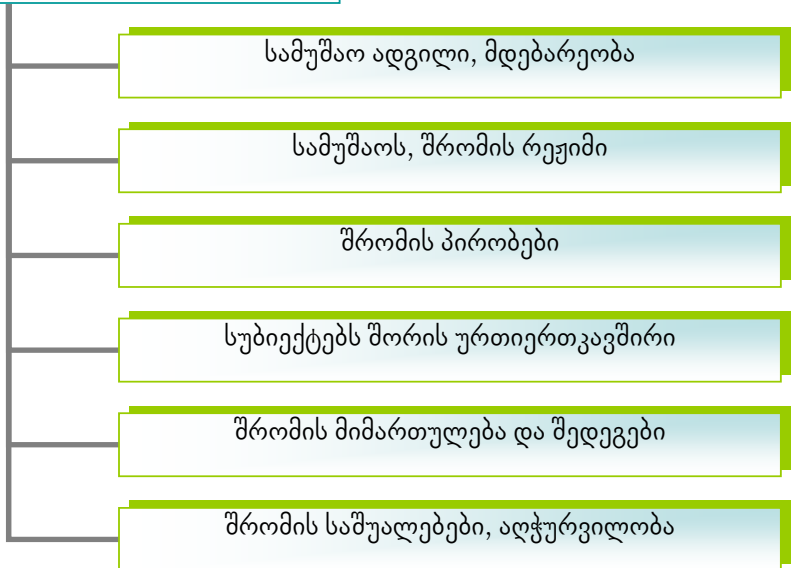

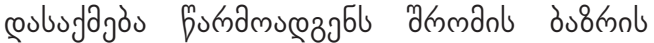

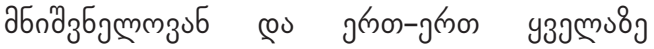

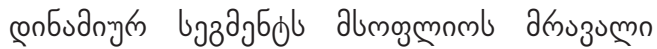

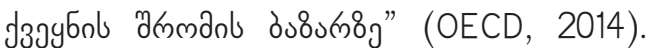

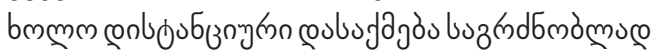

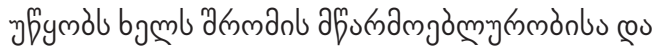

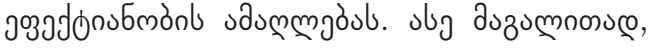

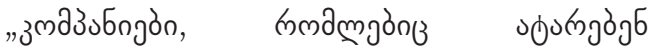

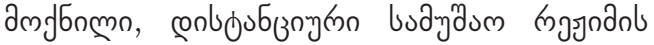

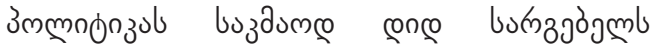

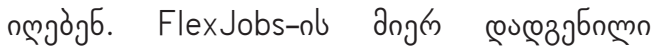

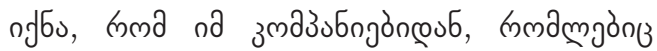

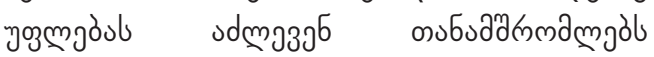

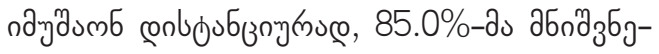

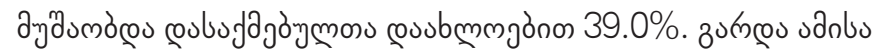

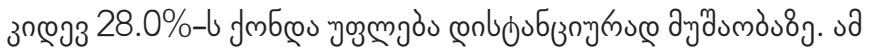

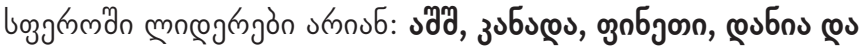

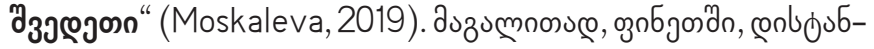

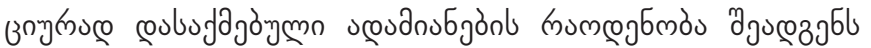

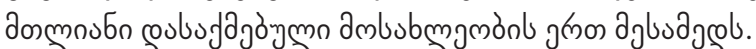

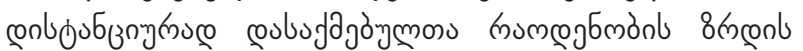

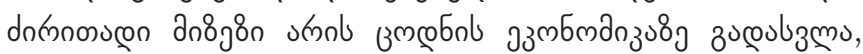

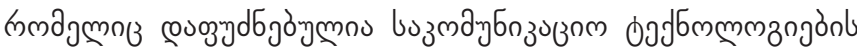

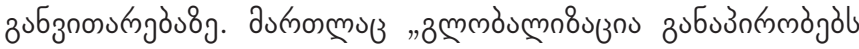

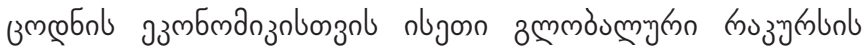

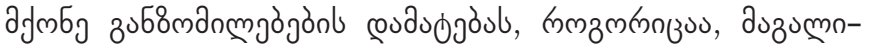

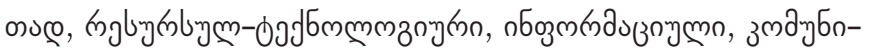

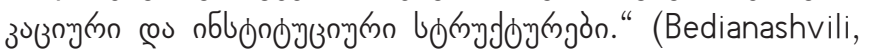

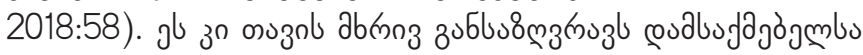

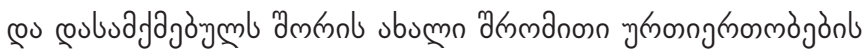

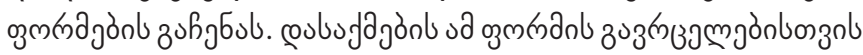

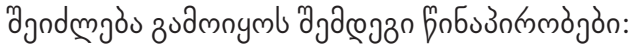

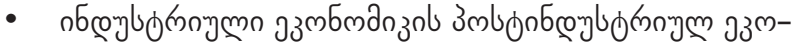

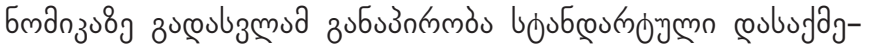

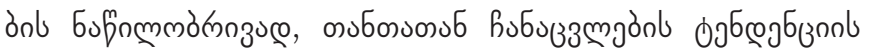

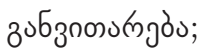

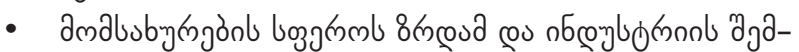

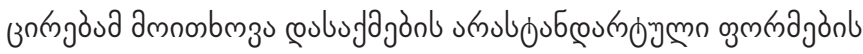

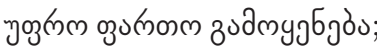

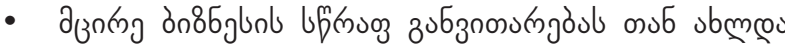

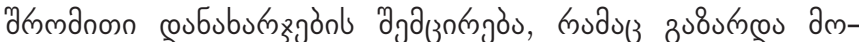

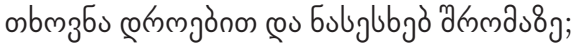

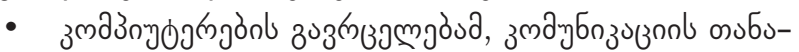

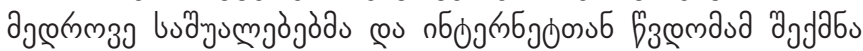

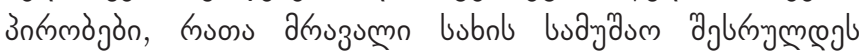

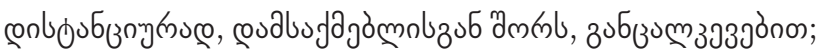

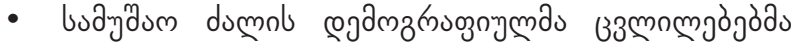

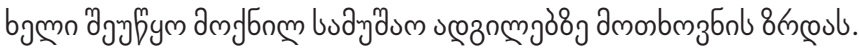

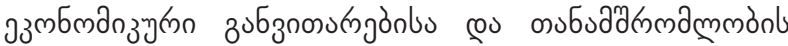

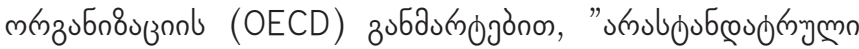

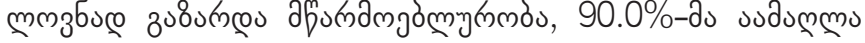

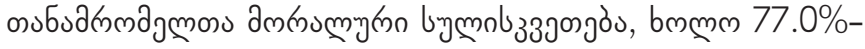

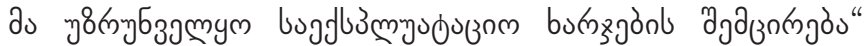

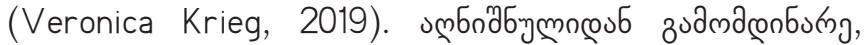

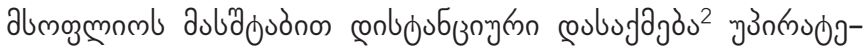

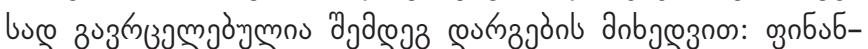

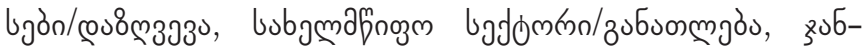

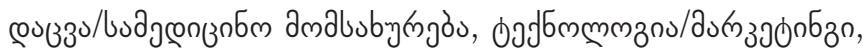

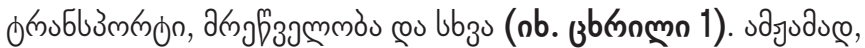

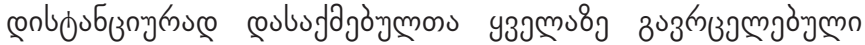

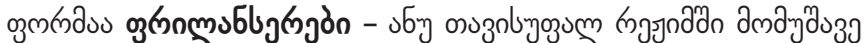

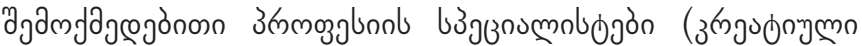

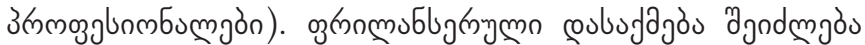

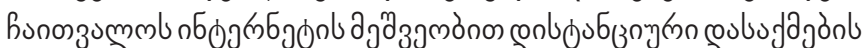

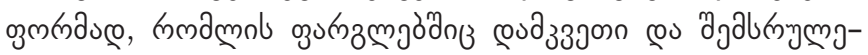

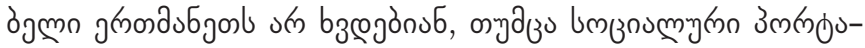

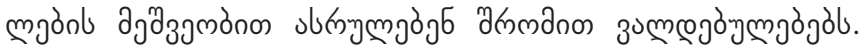

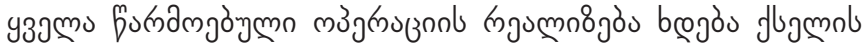

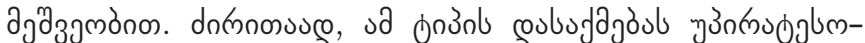

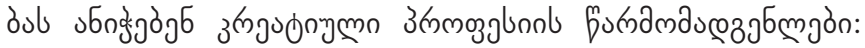

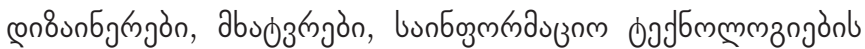

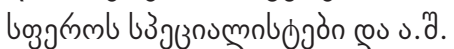

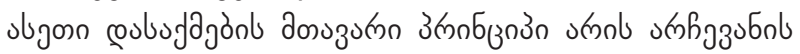

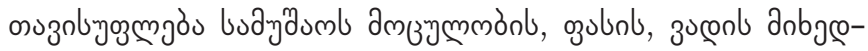

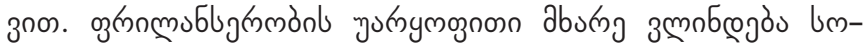

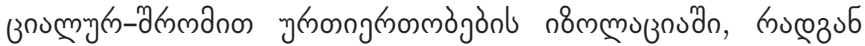

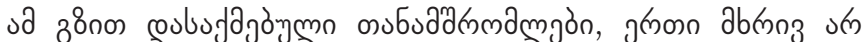

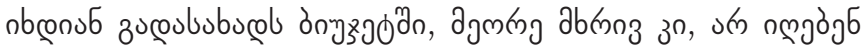

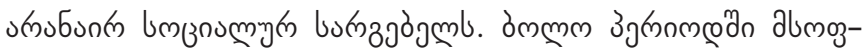

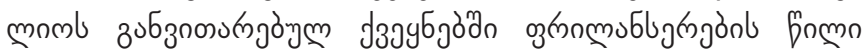

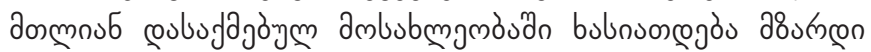

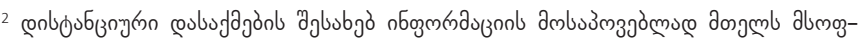

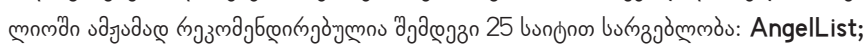
Authentic Jobs; Avon; Career Builder; Dribble; Elance; Fiverr; FlexJobs; Freelancermap.com; Guru; Indeed; Idealist; Monster; oDesk; PowerToFly; Ruby Now; SkillBridge; Skip The Drive; Stack Overflow; Staff.com; Stella \& Dot; The Muse; Virtual Vocations; We Work Remotely; Working Nomads;
} 


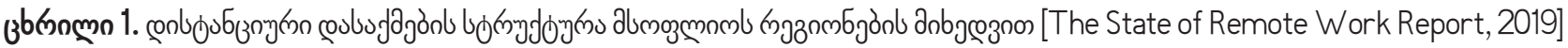

\begin{tabular}{|c|c|c|c|c|c|c|c|}
\hline 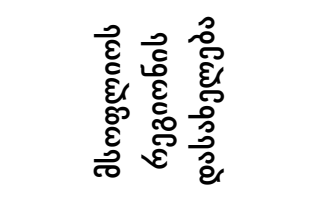 & 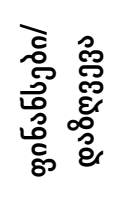 & 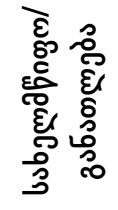 & 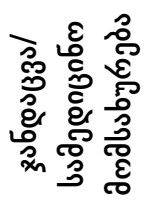 & 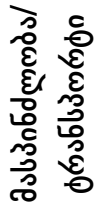 & 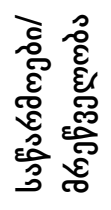 & 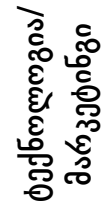 & :్ర్తె \\
\hline गoुलñus & $17.0 \%$ & $17.0 \%$ & $9.0 \%$ & $7.0 \%$ & $10.0 \%$ & $16.0 \%$ & $24.0 \%$ \\
\hline 3800 & $16.0 \%$ & $19.0 \%$ & $9.0 \%$ & $5.0 \%$ & $10.0 \%$ & $16.0 \%$ & $25.0 \%$ \\
\hline şbd mumns & $10.0 \%$ & $24.0 \%$ & $10.0 \%$ & $9.0 \%$ & $10.0 \%$ & $10.0 \%$ & $27.0 \%$ \\
\hline 53 & $13.0 \%$ & $20.0 \%$ & $11.0 \%$ & $6.0 \%$ & $13.0 \%$ & $8.0 \%$ & $29.0 \%$ \\
\hline 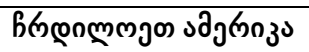 & $10.0 \%$ & $27.0 \%$ & $10.0 \%$ & $4.0 \%$ & $12.0 \%$ & $8.0 \%$ & $29.0 \%$ \\
\hline 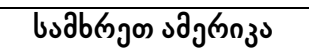 & $8.0 \%$ & $27.0 \%$ & $10.0 \%$ & $5.0 \%$ & $11.0 \%$ & $10.0 \%$ & $33.0 \%$ \\
\hline
\end{tabular}

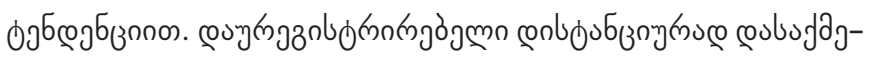

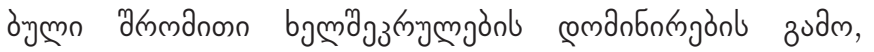

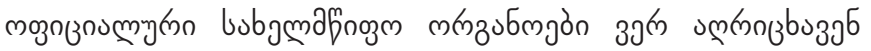

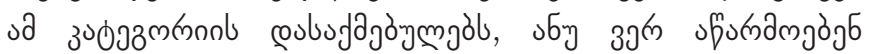

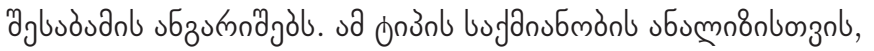

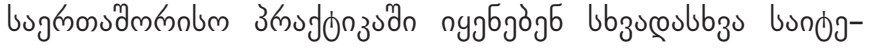

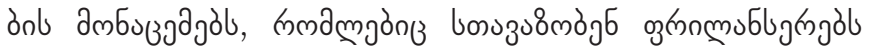

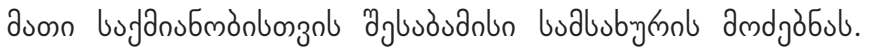

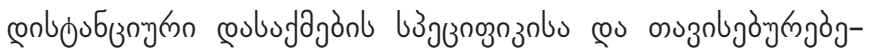

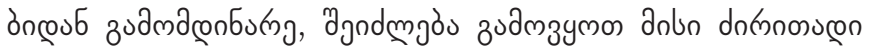

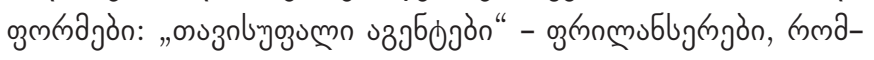

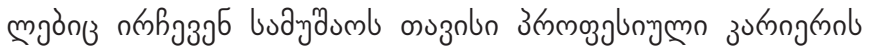

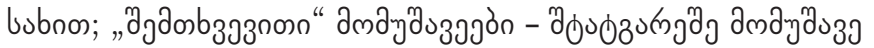

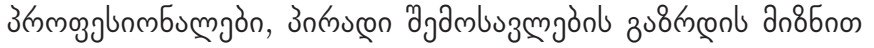

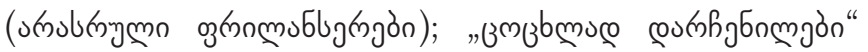

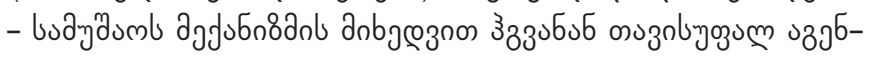

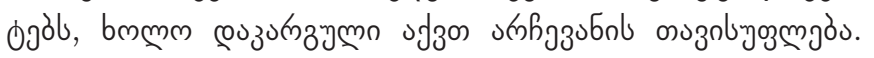

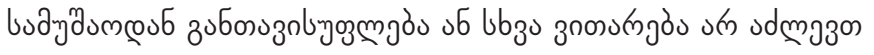

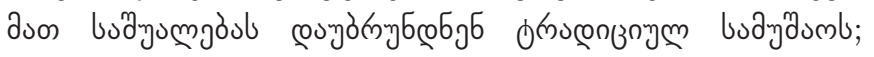

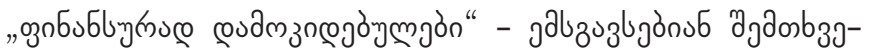

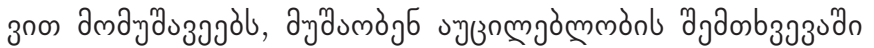

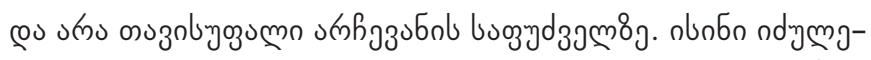

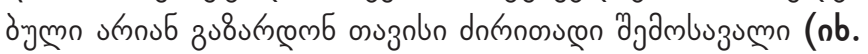

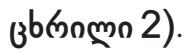

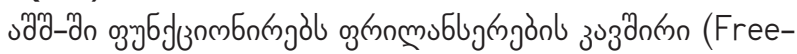

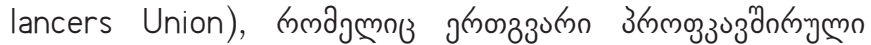

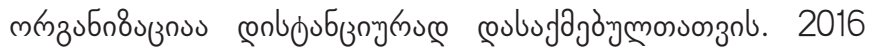

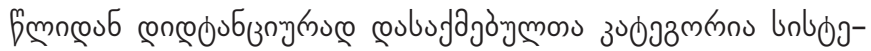

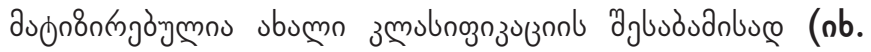
(b)

„FlexJobs eu Global Workplace Analytics-nl angkn

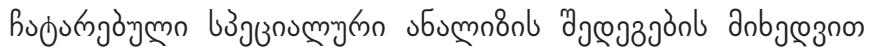

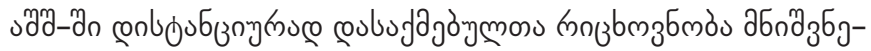

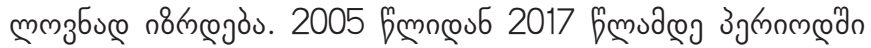

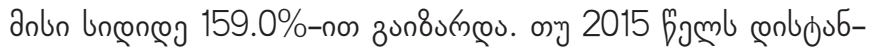

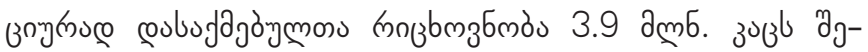

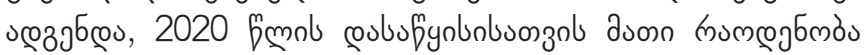

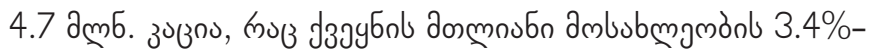

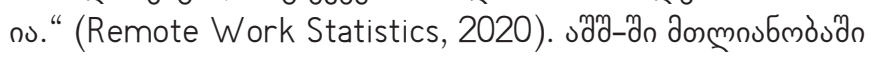

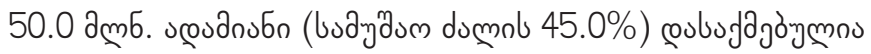

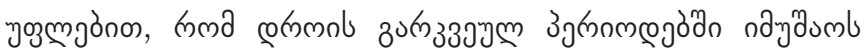

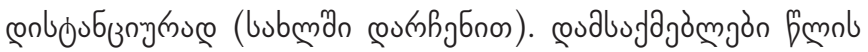

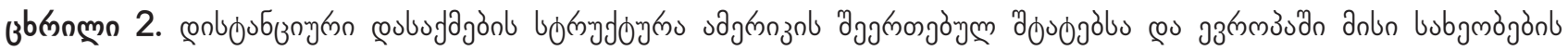

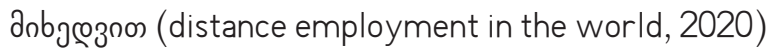

\begin{tabular}{|c|c|c|}
\hline 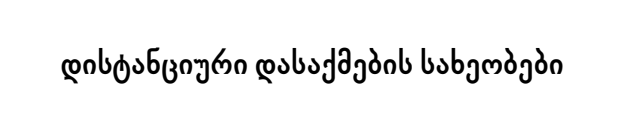 & 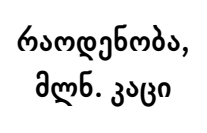 & 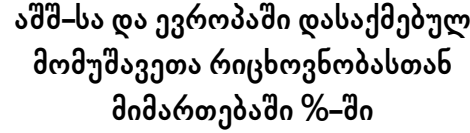 \\
\hline 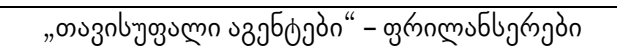 & 49.0 & $30.0 \%$ \\
\hline 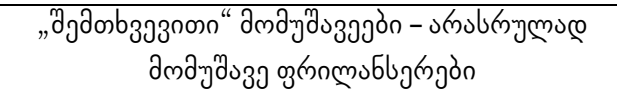 & 64.0 & $40.0 \%$ \\
\hline 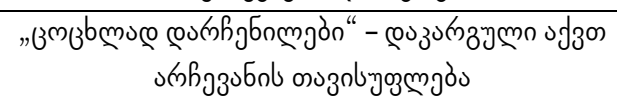 & 23.0 & $14.0 \%$ \\
\hline 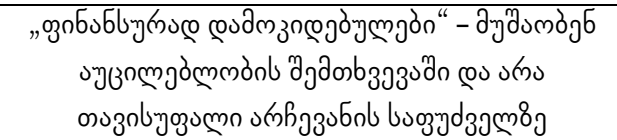 & 26.0 & $16.0 \%$ \\
\hline bym & 162.0 & $100.0 \%$ \\
\hline
\end{tabular}




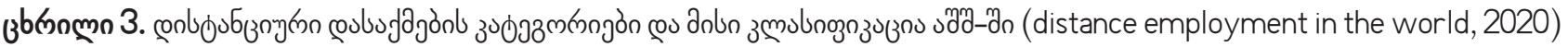

\begin{tabular}{|c|c|}
\hline 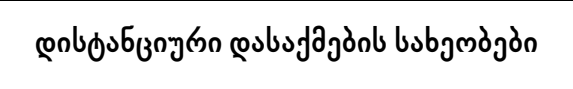 & 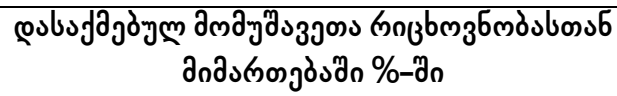 \\
\hline 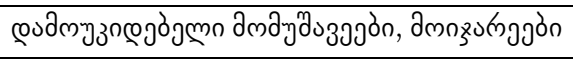 & $40.0 \%$ \\
\hline 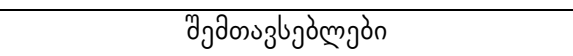 & $27.0 \%$ \\
\hline 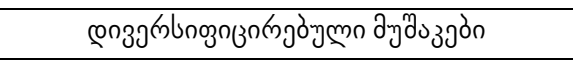 & $18.0 \%$ \\
\hline 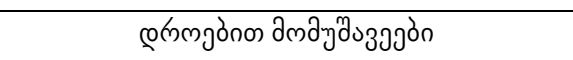 & $10.0 \%$ \\
\hline 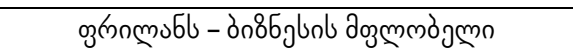 & $5.0 \%$ \\
\hline
\end{tabular}

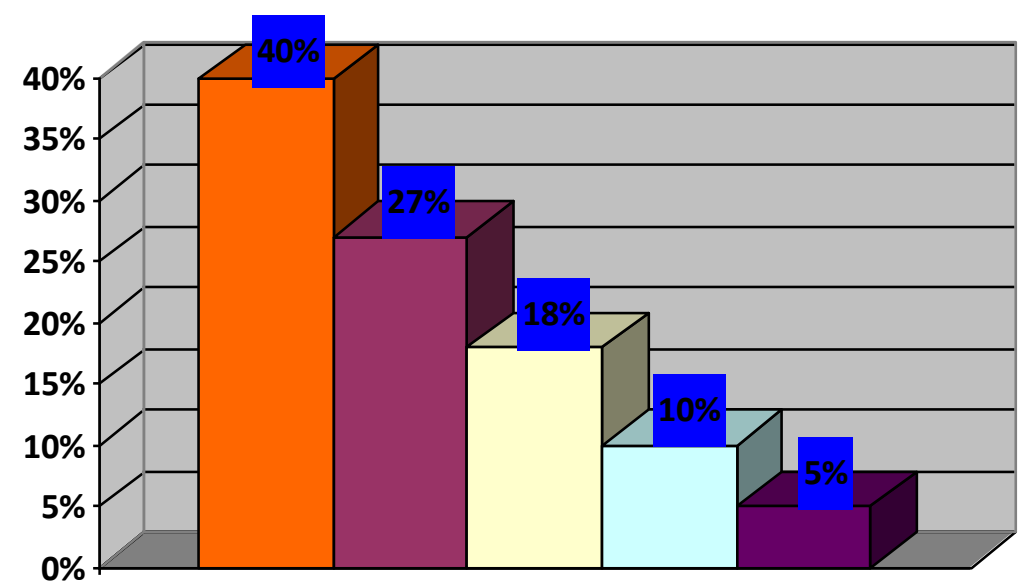

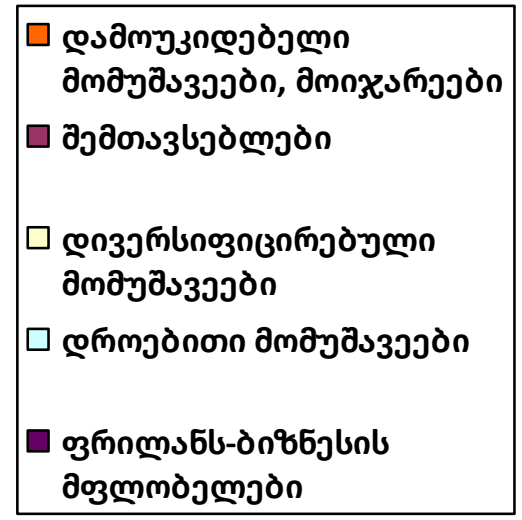

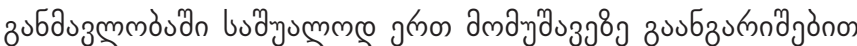

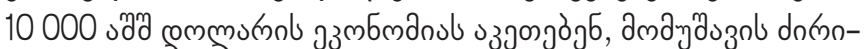

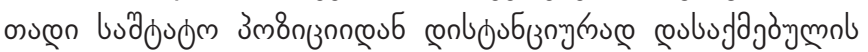

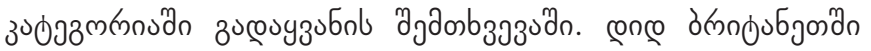

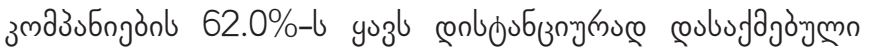

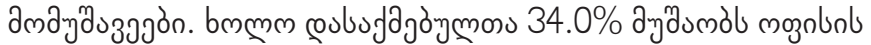

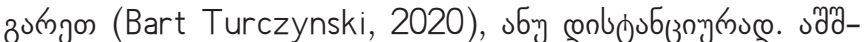

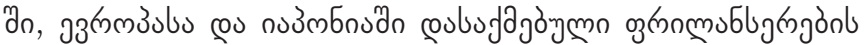

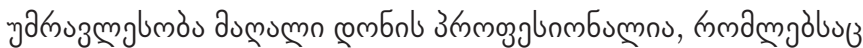

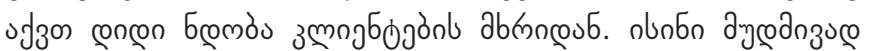

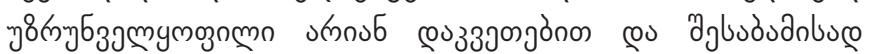

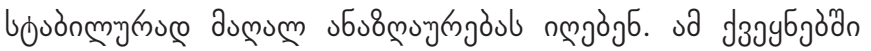

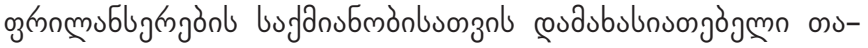

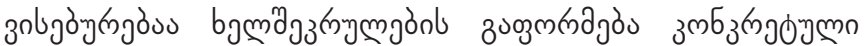

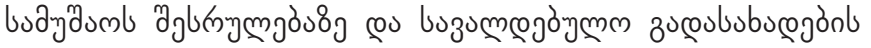

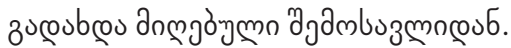

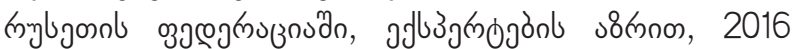

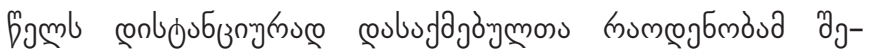

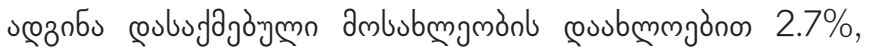

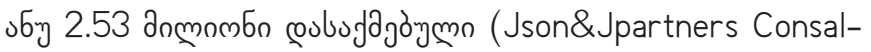

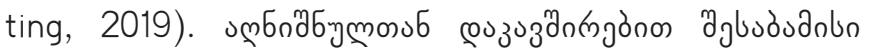

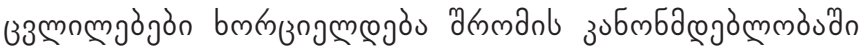

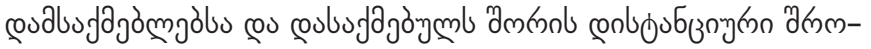

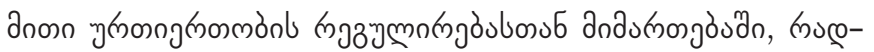

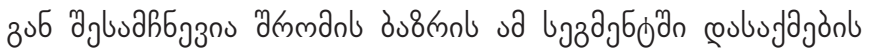

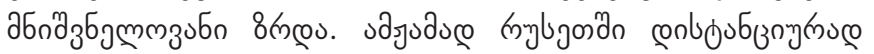

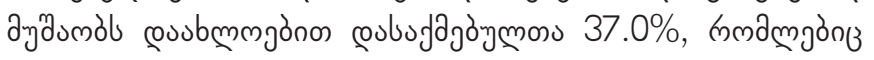

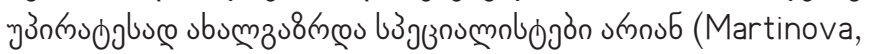

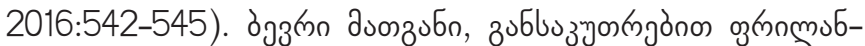

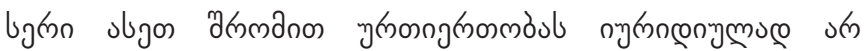

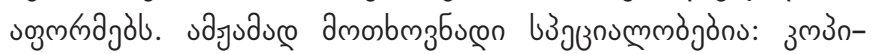

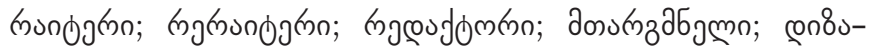

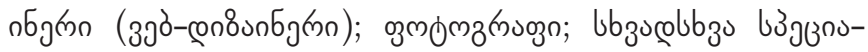

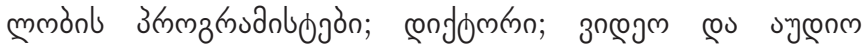

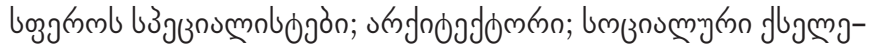

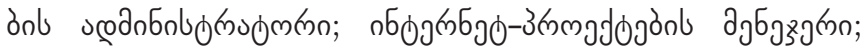

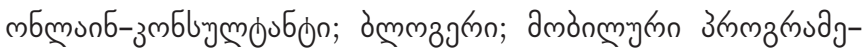

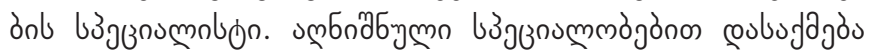

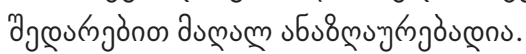

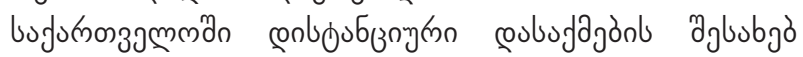

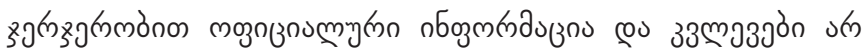

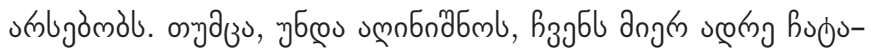

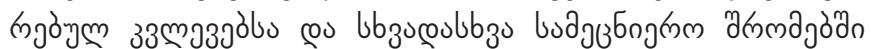
(Tsartsidze, 2019:82-100); (Tsartsidze, 2019:16-37); (Tsartsidze..., 2017:582-588) skrugnnoz8obus bu8zubanon

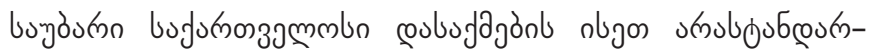

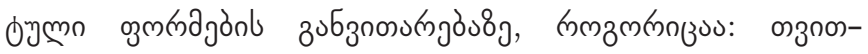

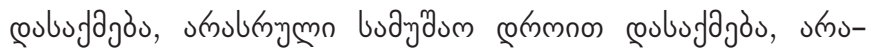

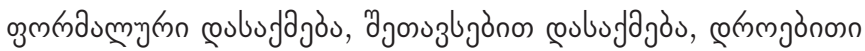

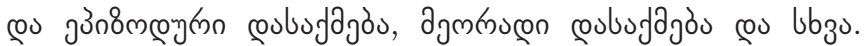

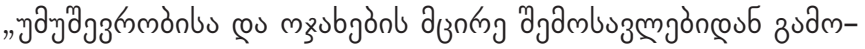

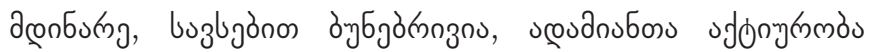

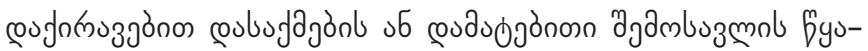

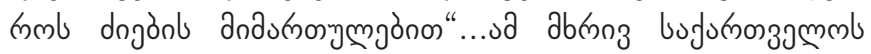

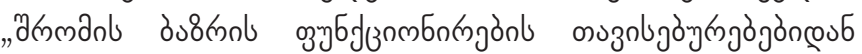

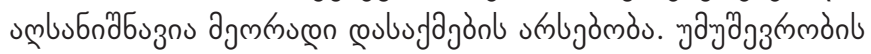




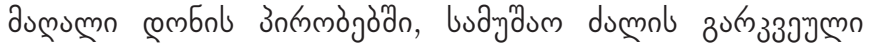

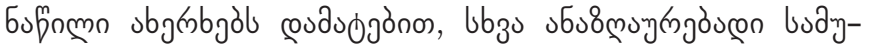
gumb gybringmobubus" (Tukhashvili,..., 2012:37,130).

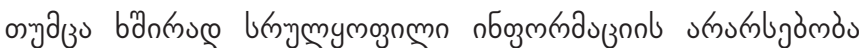

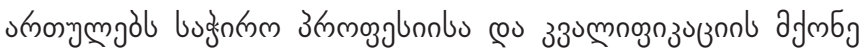

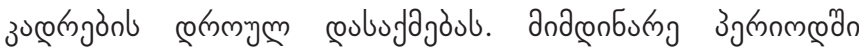

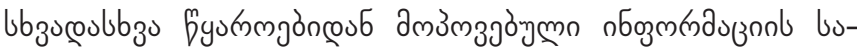

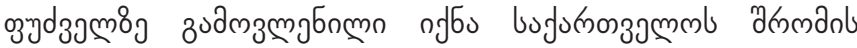

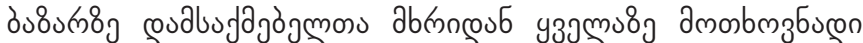

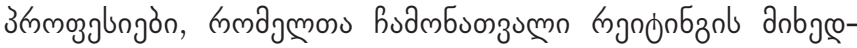

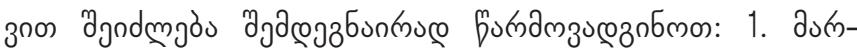

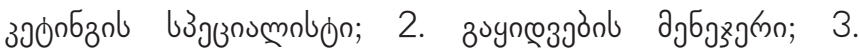

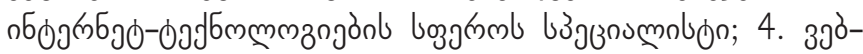

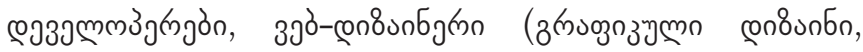

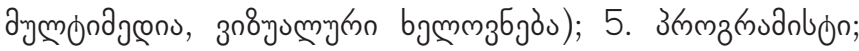

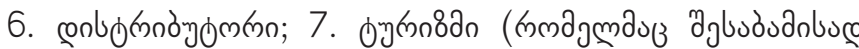

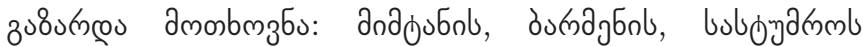

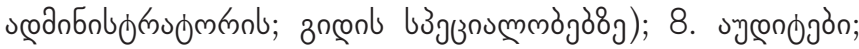

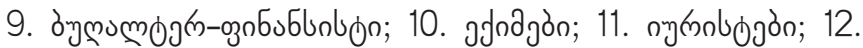

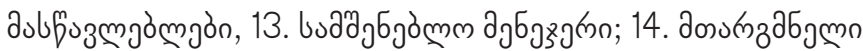

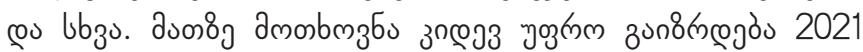

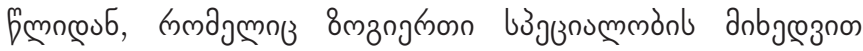

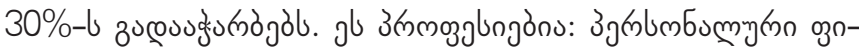

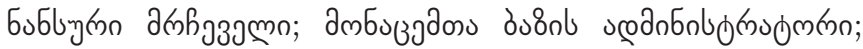

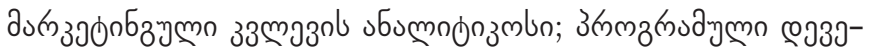

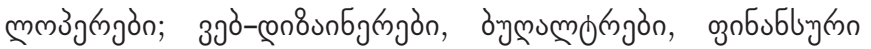

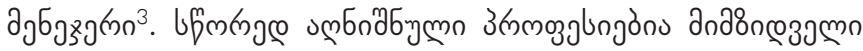

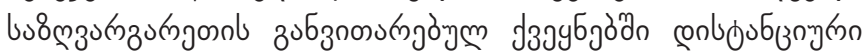

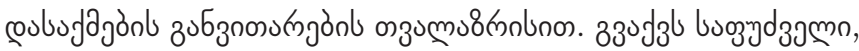

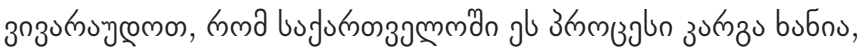

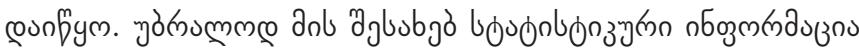

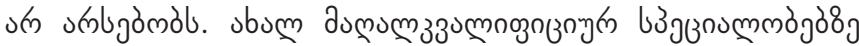

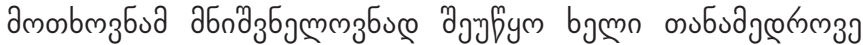

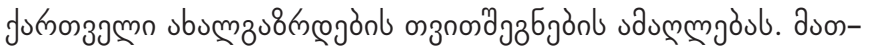

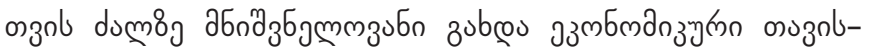

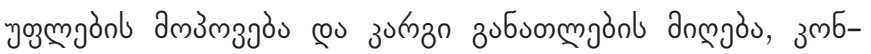

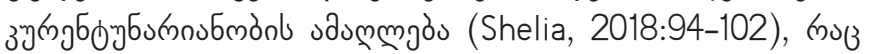

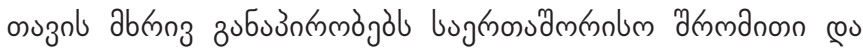

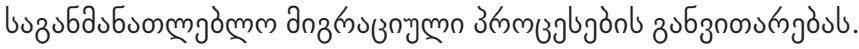

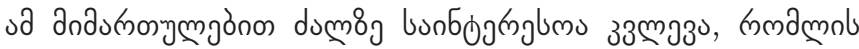

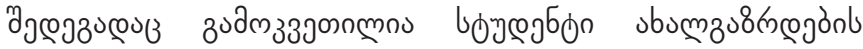

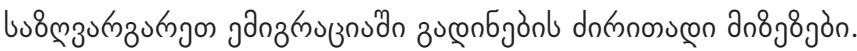

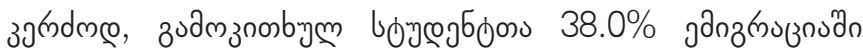

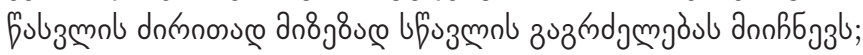

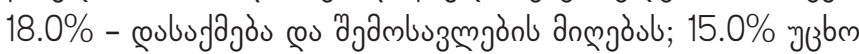

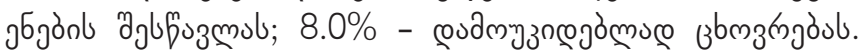

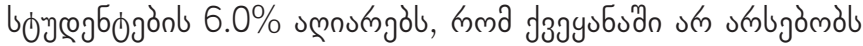

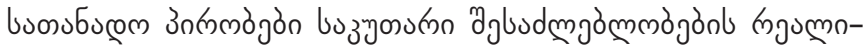

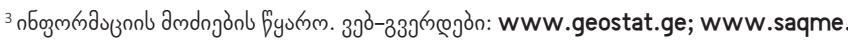
ge, www.myjobs.ge, www. hr.ge; www.jobs.ge; http://geoeconomics.ge/;

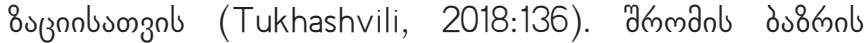

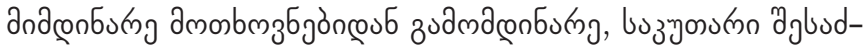

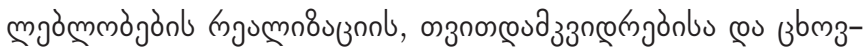

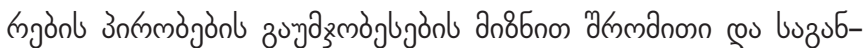

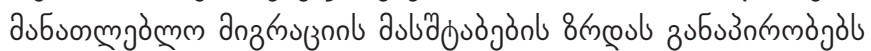

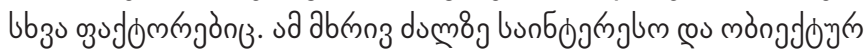

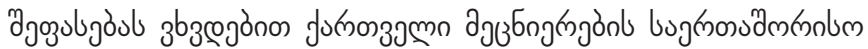

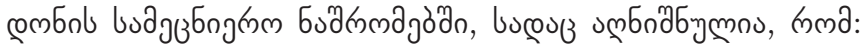

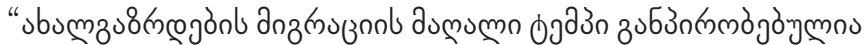

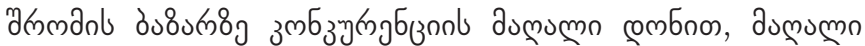

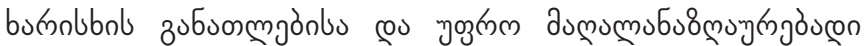

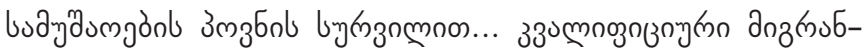

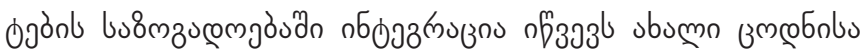

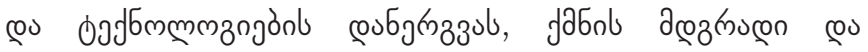

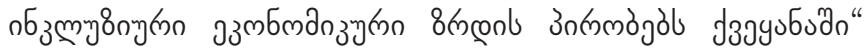

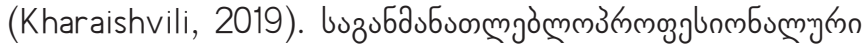

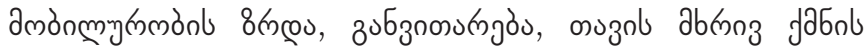

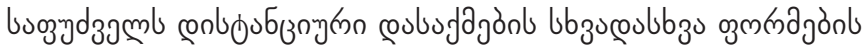

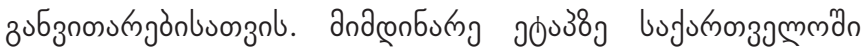

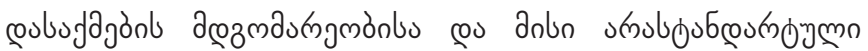

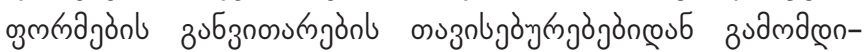

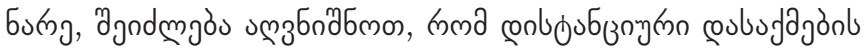

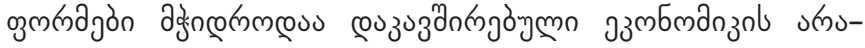

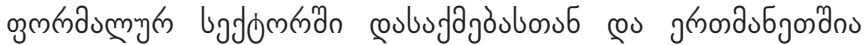

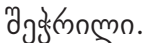

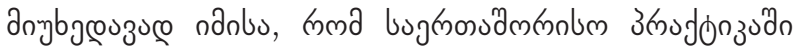

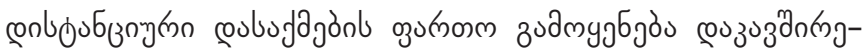

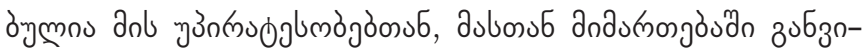

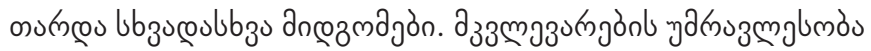

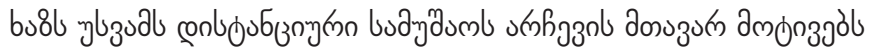

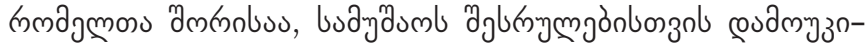

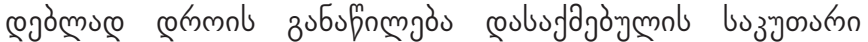
उஙnnm mnd

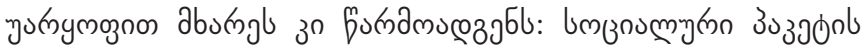

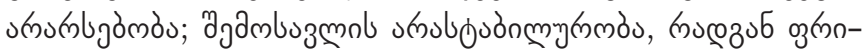

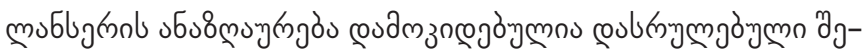

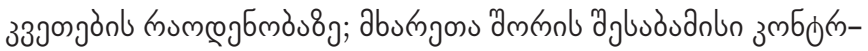

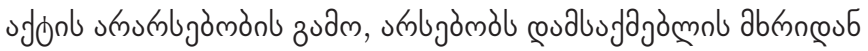

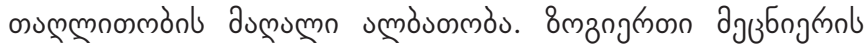

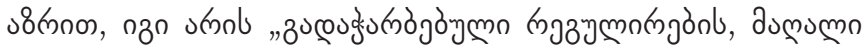

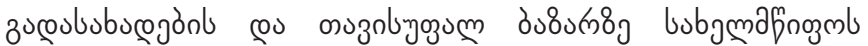

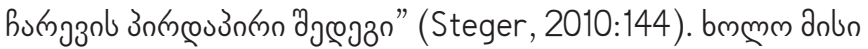

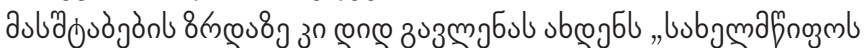

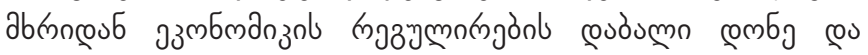

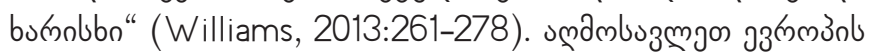

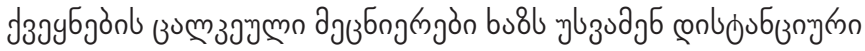

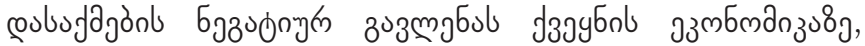

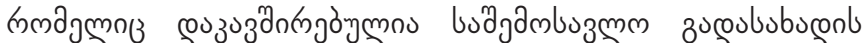

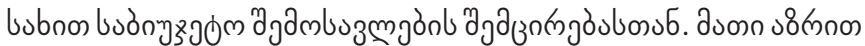

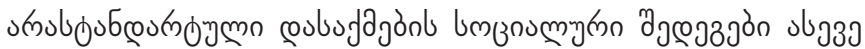




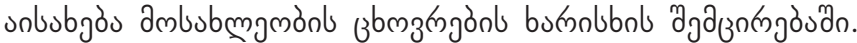

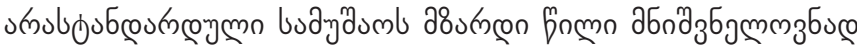

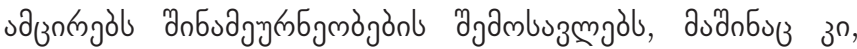

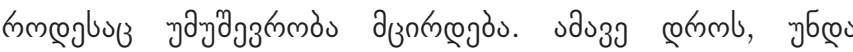

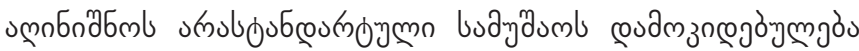

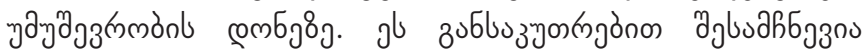

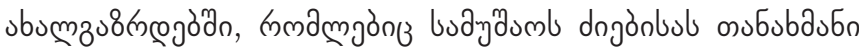

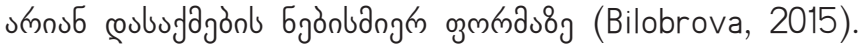

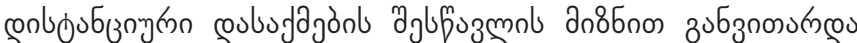

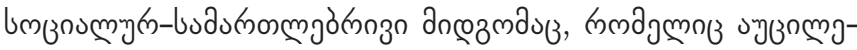

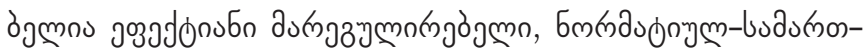

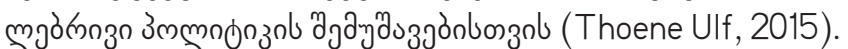

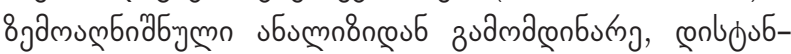

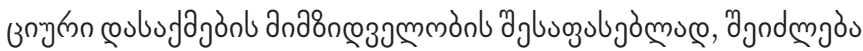

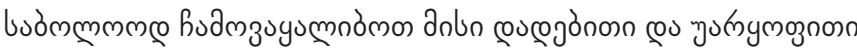

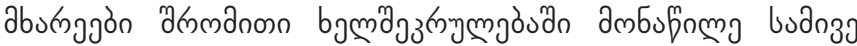

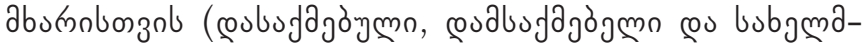

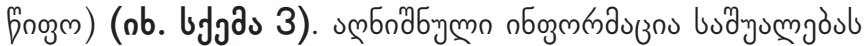

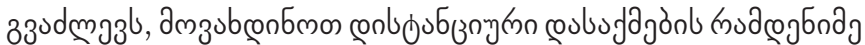

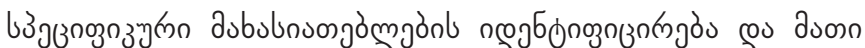

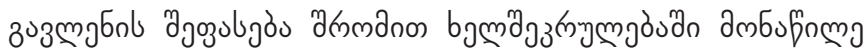
aburnggobog.

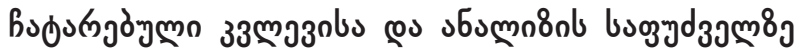

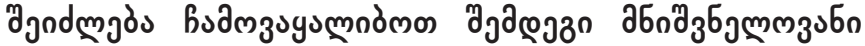

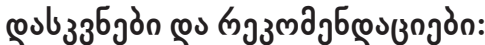

1. mubuajon

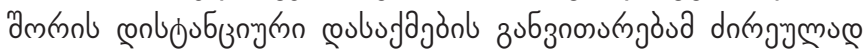

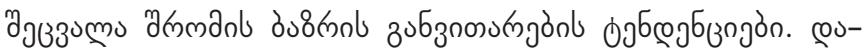
bujajön u u

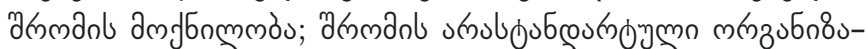

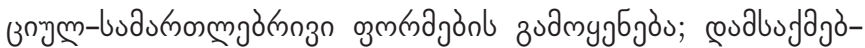

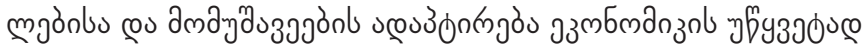

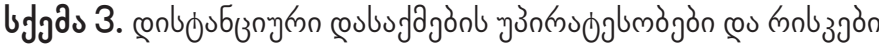

\begin{tabular}{|c|c|c|}
\hline 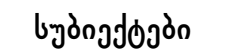 & 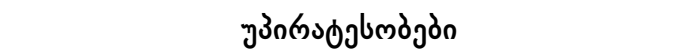 & molzjodn \\
\hline \multirow{8}{*}{ 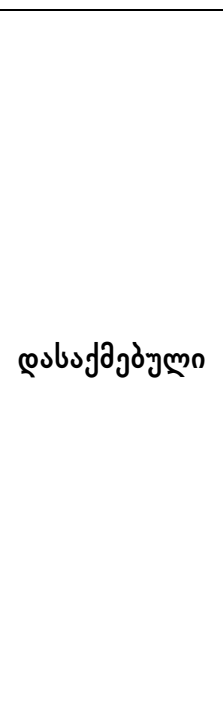 } & 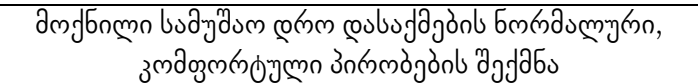 & 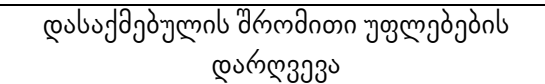 \\
\hline & 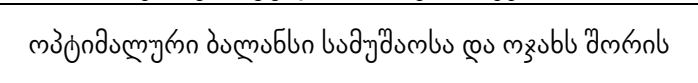 & 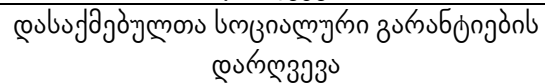 \\
\hline & 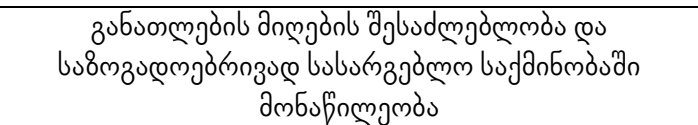 & 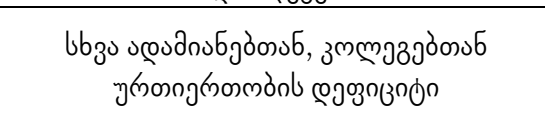 \\
\hline & 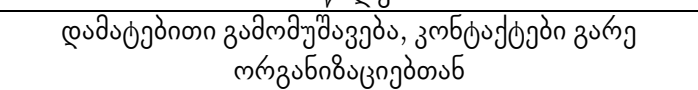 & 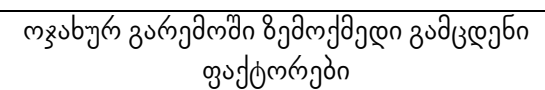 \\
\hline & 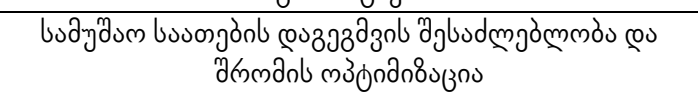 & 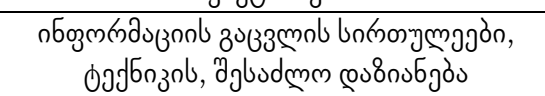 \\
\hline & 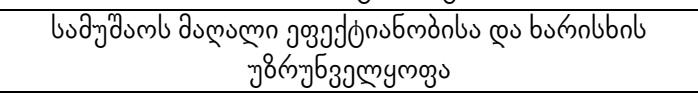 & 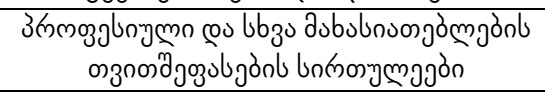 \\
\hline & 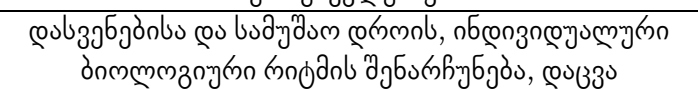 & \multirow{2}{*}{ 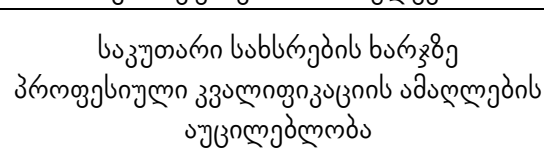 } \\
\hline & 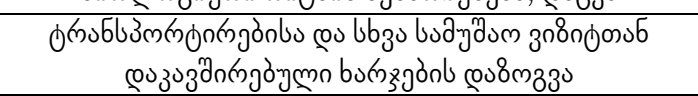 & \\
\hline \multirow{5}{*}{ 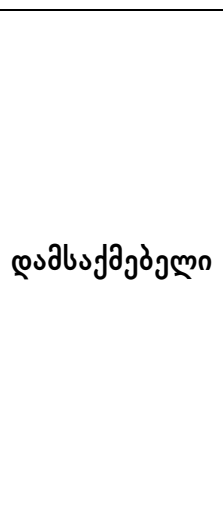 } & 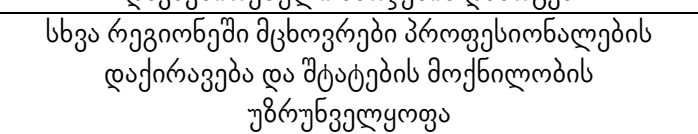 & 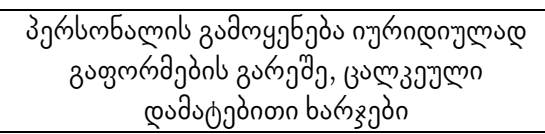 \\
\hline & 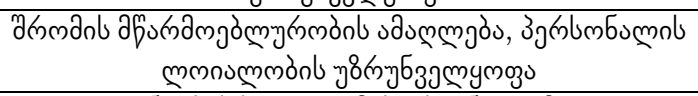 & 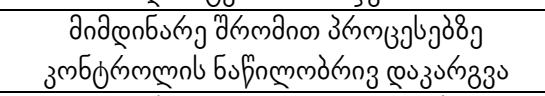 \\
\hline & 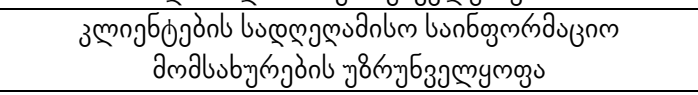 & 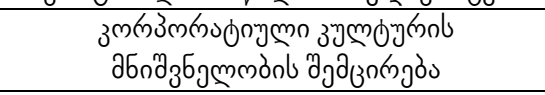 \\
\hline & 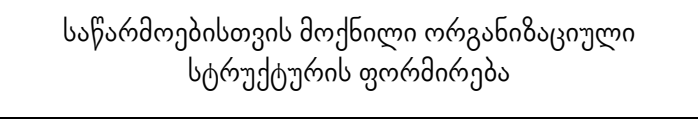 & \multirow{2}{*}{ 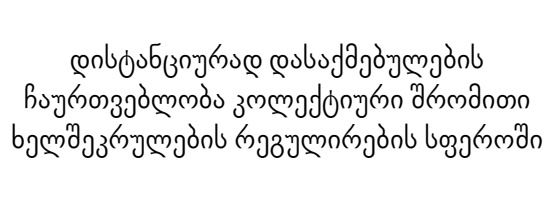 } \\
\hline & 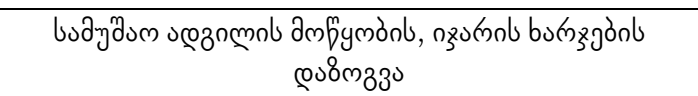 & \\
\hline \multirow{3}{*}{ bsbэмдрповм } & 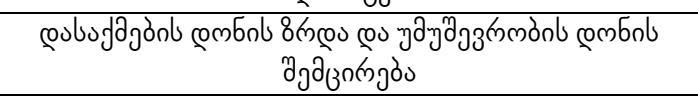 & 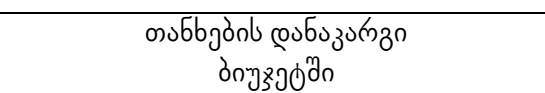 \\
\hline & 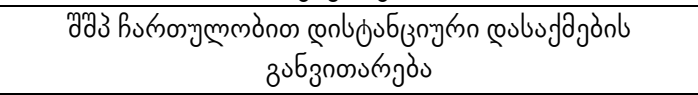 & \multirow{2}{*}{ 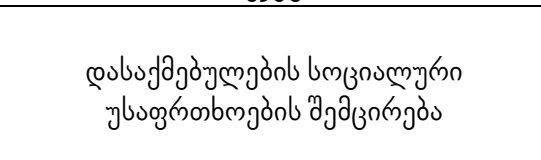 } \\
\hline & 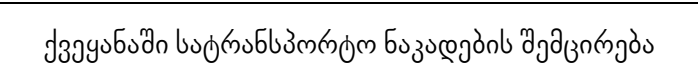 & \\
\hline
\end{tabular}




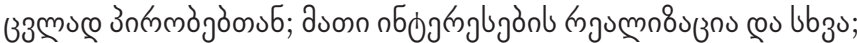

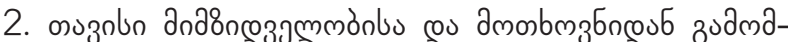

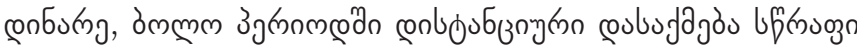

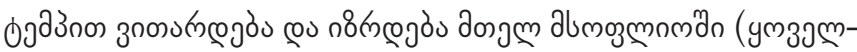

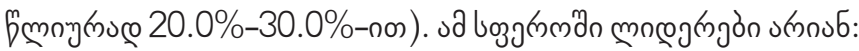

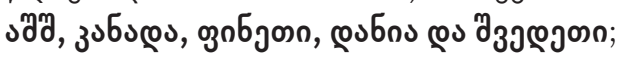

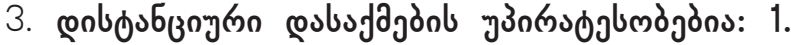

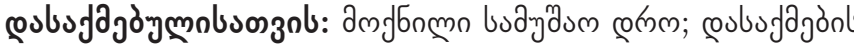

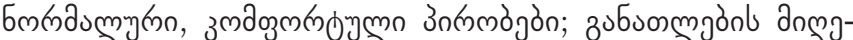

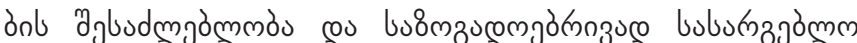

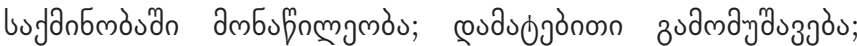

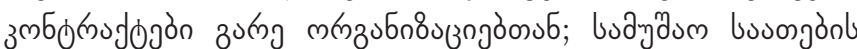

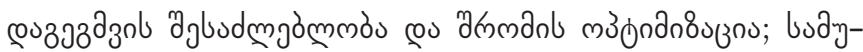

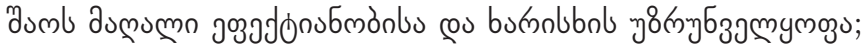

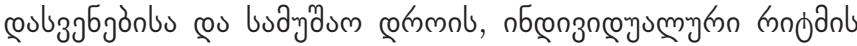

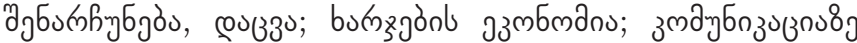

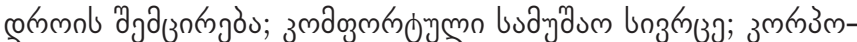

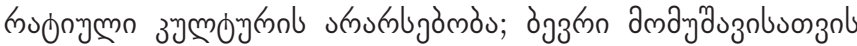

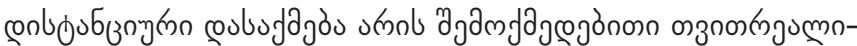

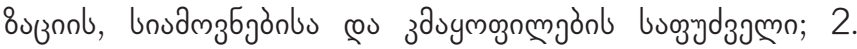

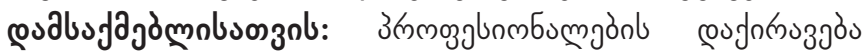

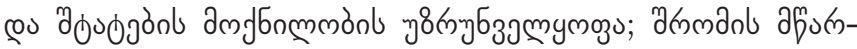

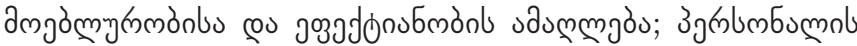

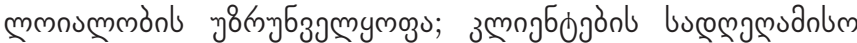

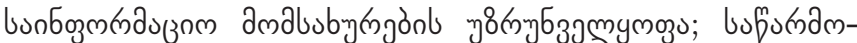

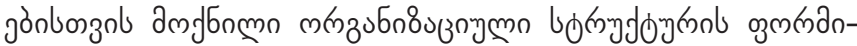

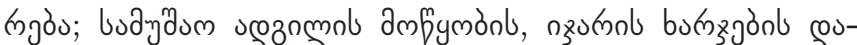

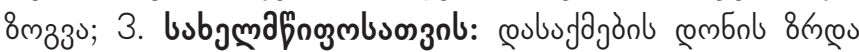

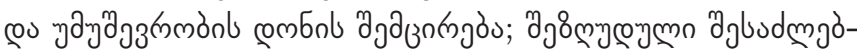

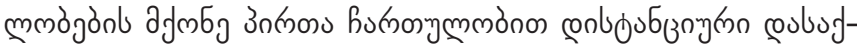

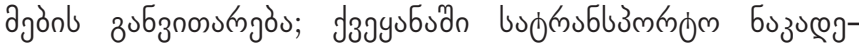

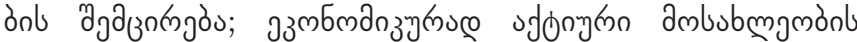

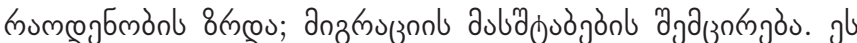

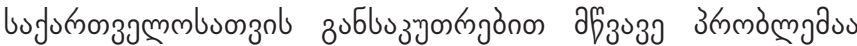

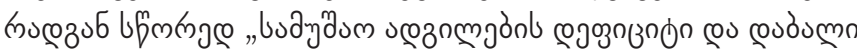

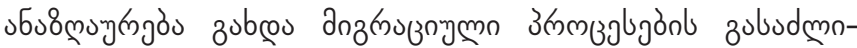

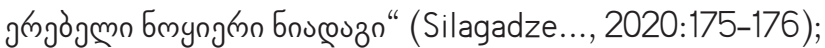

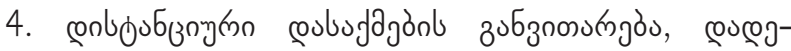

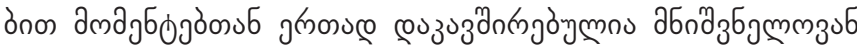

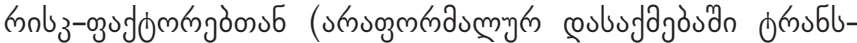

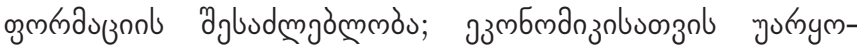

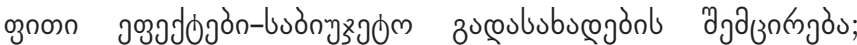

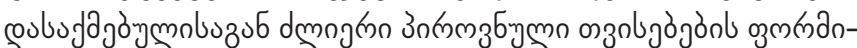

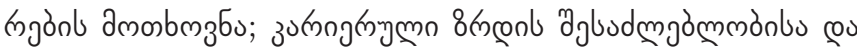

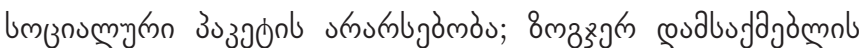

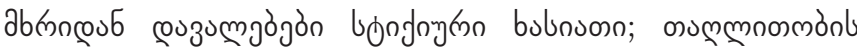

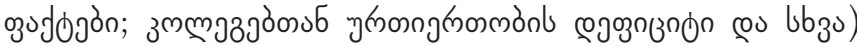

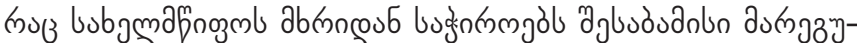

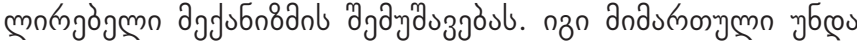

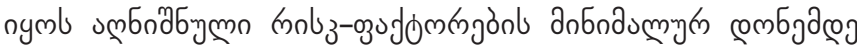

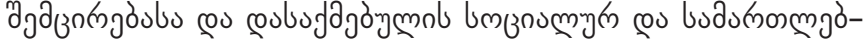

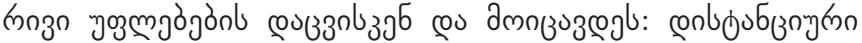

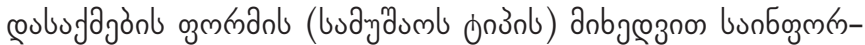

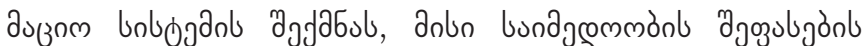

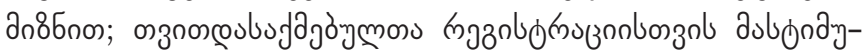

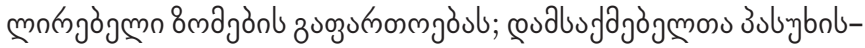

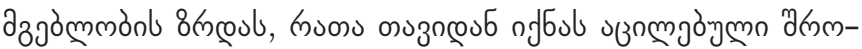

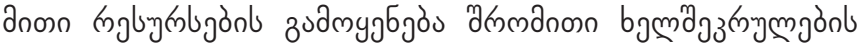

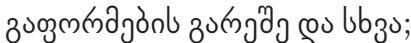

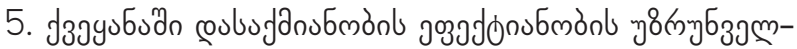

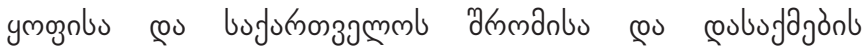

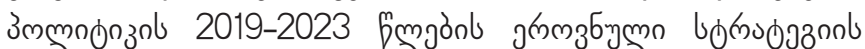

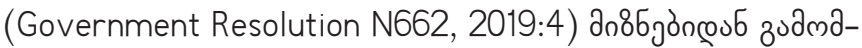

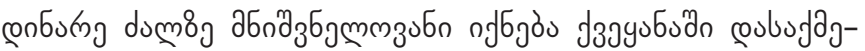

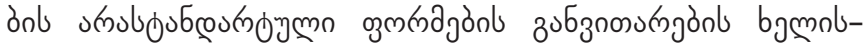

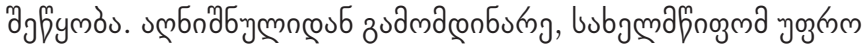

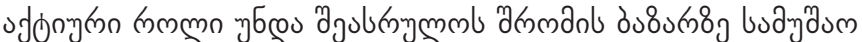

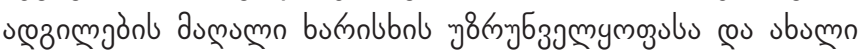

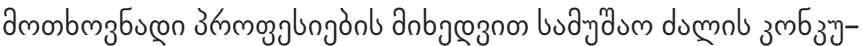

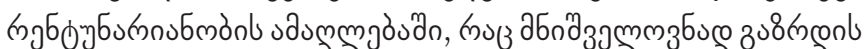

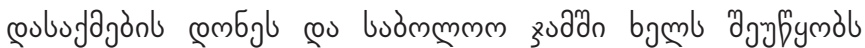

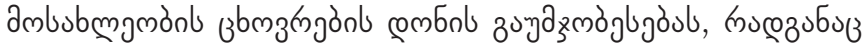

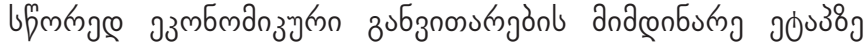

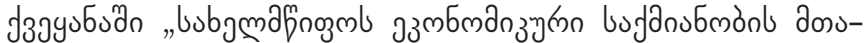

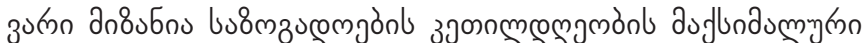
3ง8ำou" (Mekvabishvili, 2019:22-23);

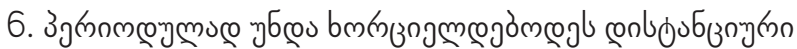

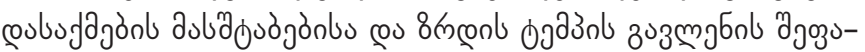

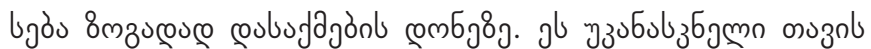

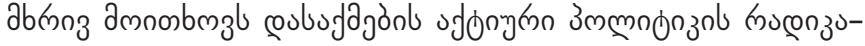

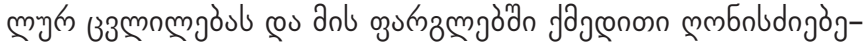

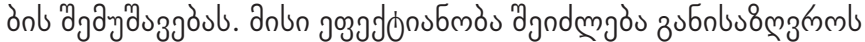

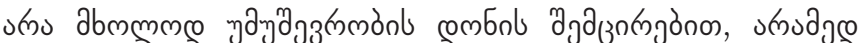

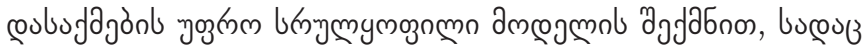

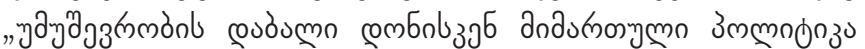

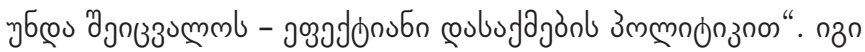

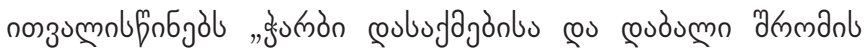

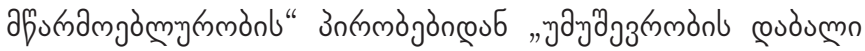

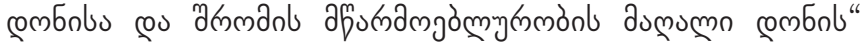

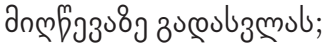

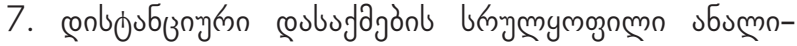

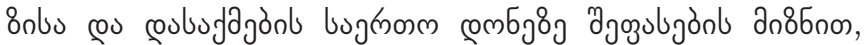

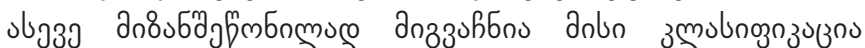

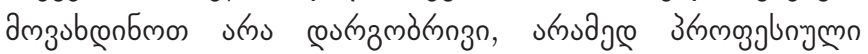

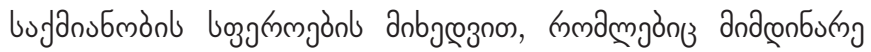

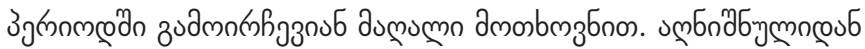

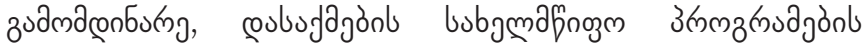

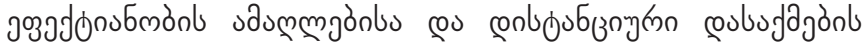

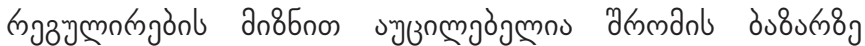

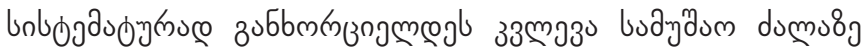




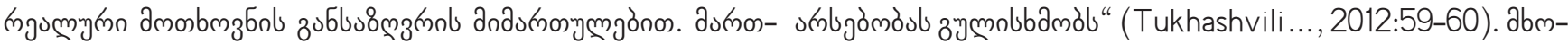

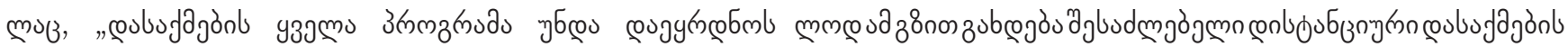

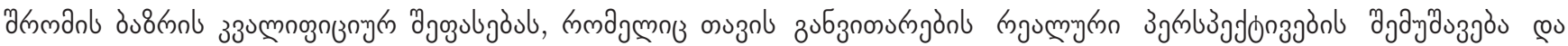

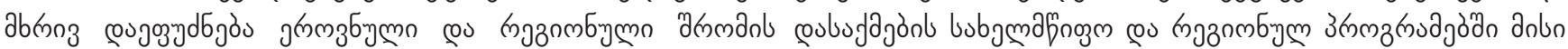

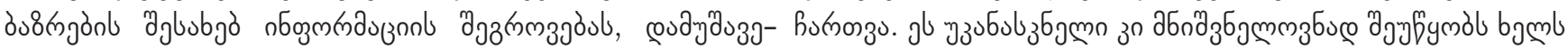

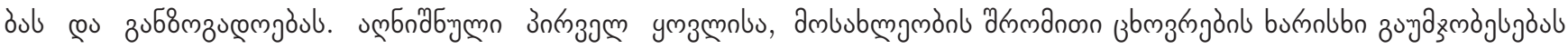

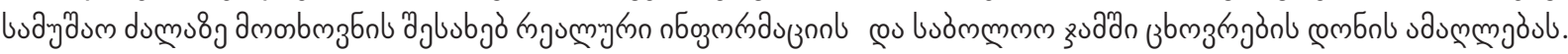

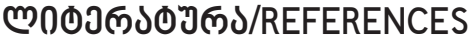

Bedianashvili, G., (2018). Culture as a factor in the formation of the knowledge economy in a systemic institutional context of paradigm shift. Globalization and Business, 6, Tbilisi. 2449-2396. http://www.eugb.ge/uploads/content/N6/GiviBedianashvili.pdf; (In Georgian)

Mekvabishvili, E., (2019). The economic role of the state in the context of globalization. Globalization and Business, 8; https://www.eugb.ge/ view_content.php?content=content\&id=228\&THE\%20ECONOMIC\%20ROLE\%200F\%20THE\%20 STATE\%20IN\%20CONDITIONS\%200F\%20GLOBALIZATION; (In Georgian)

Papava, V., \& Charaia, V., (2020). The Coronomic Crisis and Some Challenges of The Georgian Economy.Expert Opinion. Georgian Strategy and International Relations Research Foundation Tbilisi. HTTPS://WWW.GFSIS.ORG/FILES/LIBRARY/ OPINION- PAPERS/136-EXPERT-OPINION-GEO.PDF; (In Georgian)

Papava, V., (2015). Economy of Georgia. Reforms and Pseudo-Reforms. Publishing Intellect. Tbilisi; (In Georgian).

Tukhashvili, M., Ananiashvili I., Tsartsidze M., Latsabidze N., Shelia m., \& Chelidze N. (2012) Labor Market and Reintegration of Returned Migrants in Georgia. The publication was prepared with the support of the Danish Refugee Council project «Reintegration Activities in Georgia». Universal Publishing House, Tbilisi. (In Georgian)

Gaganidze, G., (2018). Expert Aspects of Country Competitiveness Assessment. Globalization and Business, 6, European University, Tbilisi; 2449-2396. http://www.eugb.ge/uploads/content/N6/Giorgi-Gaganidze.pdf; (In Georgian)

Tsartsidze, M., (2019). Effective employment - an important factor in human well-being and economic growth in Georgia. Ekonomisti, 2, e. Version; EISSN 2346-8432Print ISSN 1987-6890. http://ekonomisti.tsu.ge/?cat=nomer\&leng=ge; (In Georgian)

Tsartsidze, M., (2019). Active labor market policy and peculiarities of youth employment in Georgia. Economics and Business, XI, 2. ISSN 1987-5789. 16-37. http://eb.tsu.ge/?leng=ge. (In Georgian)

Tsartsidze, M., \& Latsabidze, N. (2017). Unemployment and active employment policy at the current stage of labor market formation in Georgia. II International Scientific Conference - Challenges of Globalization in Economics and Business. TSU Faculty of Economics and Business. Proceedings. Tbilisi; ISBN 978-3-9941-13-650-4. https://www.tsu.ge/data/file_db/ economist_faculty/global.pdf. (In Georgian)

Resolution of the Government of Georgia, 662, On Approval of the National Strategy of Labor and Employment Policy of Georgia for 2019-2023, Tbilisi, December 30, 2019. (In Georgian)

Organic Law of Georgia N4113, Labor Code of Georgia, Tbilisi, December 17, 2010; (In Georgian)

Bilobrova, T., \& Tul S. Jobless society - phenomenon of global economy. Economic Processes Management. http://nbuv.gov. ua/UJRN/EPM_2015_3_9.

Turczynski, B., (2020). 2020 HR Statistics: Job Search, Hiring, Recruiting \& Interviews. The definitive list of human resource statistics and research for 2020. See data on resumes, job interviews as well as hiring and recruiting. https://zety.com/ blog/hr-statistics?gclid=EAlalQobChMI-Pu10Y6G6QIVxeN3Ch0gYwsnEAAYAS AAEgKDVvD_BwE.

Chreneková, M., Melichová, K., Marišová, E., \& Moroz S. (2016). Informal Employment and Quality of Life in Rural Areas of Ukraine. European Countryside.8 (2):135-146.

Fluter-Hoffman, C., \& Solbrig J. (2003). Arbeitszeitflexibilisierung: Erfolgskonzept auch fur kleine und mittlere Unternehmen. Koln,; https://www.iwkoeln.de/fileadmin/ publikationen/2003/53867/trends02_03_3.pdf.

Dorsam, P., (1997). Flexible Arbeitszeitgestaltung in mittelstandischen Unternehmen. Stuttgart: Schaffer-Poeschel. https:// books.google.ge/books.

Json\&Jpartners Consalting: http://json.tv/ict_telecom_analytics_view/polnaya-versiya-prezentatsii-generalnogo-direktorasvetlanyvodyanovoy-s-press-konferentsii-bitriks24-ijson-partnersconsulting20150617030352.

Kharaishvili E, Natsvlishvili I. (2019). Impact of International Educational Migration in The Contexst Of Globalization of Higher Education (Case Of Georgia). https://iated.org/concrete3/paper_detail.php? paper_id=73405. ISBN: 978-84-09-120314.

OECD (2014). Employment Review. http://www.oecd.org/els/employmentoutlook-previouseditions.htm.

Pisica C., Vasile V., Voineagu V. (2012). The labor market between formal and informal, Expert Publishing House. (In Romanian) Remote Work Statistics: Shifting Norms and Expectations. February 13, 2020. https://www.flexjobs. com/blog/post/remotework-statistics/. 
Steger, M., B., Neoliberalism. Oxford University Press. 144 2010. https://global.oup.com/academic/product/.

Silagadze, A., Atanelishvili T., \& Silagadze, N., (2020). Some Tendencies of Post-Soviet Migration and Unemployment. BULLETIN OF THE GEORGIAN NATIONAL ACADEMY OF SCIENCES, vol. 14, no. 1. http://science.org.ge/bnas/t14-n1/27_Silagadze_ Economis.pdf.

Shelia, M., (2018). Reproductive Behavior of Students in Georgia. Demography and social economy, 2(33). 94-102; http://dse. org.ua/arhcive/33/33_7en.html. (In Ukrainian)

Shelia M. (2014). Employers Demand on Labour Force in Georgia s Big Cities. Interdisciplinary Perspectives on Social Sciences. Cambridge Scholars Publishing, UK. 113-118.

Shelia, M., (2015). Students' attitude towards the conflict in the family. j. Humanities and Social Sciences Review, 4 (2). URL: http://www.universitypublications.net /hssr/ 0402/index.html.

Tukhashvili, M., (2012). Georgian Labour Market in the condition of Political and Economic Crisis.In the book: Youth Employment: Challenges and Opportunites. Timisoara.Eurostampa, 294-300.

Tukhashvili, M., \& Shelia, M. (2012). The impact of labor emigration on the demographic and economic development of Georgia in the post-Soviet period. http://www.carim-east.eu/media/CARIM-East-2012-RR-29.pdf.

Tukhashvili, M., Tsartsidze M., Latsabidze, N., Shelia, M., \& Lobzhanidze M., (2018). Emigration intentions of students of higher education institutions of Georgia (Examples From Ivane Javakhishvili Tbilisi State University). European Journal of Humanities and Social Sciences. ISSN 2414-2344. http://eprints.tsu.ge/view/creators/Shelia=3AMzia=3A=3A.html.

Tsartsidze, M., (2018). Unemployment and the effective employment problems in Georgia under the modern globalization terms. Institute of Researches and International Symposiums IRIS-ALKONA. Latvia. Journal of International Economic Research. ISSN 2500-9656. Volume 4. No.1.http://irissymposium.wixsite.com/website.

Tsartsidze, M. (2018). Problems of Labor Market Formation and the Strategic Directions for its Overcomings in Georgia. Institute of Researches and International Symposiums IRIS-ALKONA. Latvia. ISSN 2414-2344. Journal of International Academic Research JIAR. ISSN 2592-8600. VOLUME-1. NO.1.http://irissymposium. wixsite.com/website-71.

Thoene, Ulf., (2015) A Socio-Legal Exploration of the Linkages between Informal Employment, Social Protection and Labour Law in Latin America. Revista de Estudios Sociales. https://journals.openedition. org/revestudsoc/9412.

The State of Remote Work Report. How employees around the world think about working remotely, hybrid and fully remote companies, how learning styles affect remote teams, and more. A report published annually by Owl Labs. https://www. owllabs.com/state-of-remote-work.

Veronica Krieg. (2019). How to Give Work from Home Call Center Agents the Support and Resources they Need to Keep Your Business Functioning at Its Best. https://www.business2community.com/strategy/how-to-give-work-from-home-callcenter-agents-the-support-and-resources-they-need-to-keep-your-business-functioning-at-its best-02294442.

Williams, C., (2013). Tackling Europe's Informal Economy: A Critical Evaluation of the Neo-liberal De-regulatory Perspective. Journal of Contemporary European Research. https://www.jcer.net/index.php/jcer/ article/ view/496

Varshavskaya, E. Ya. (2009). Employment flexibility: foreign experience and Russian practice. Kemerovo: Kemerovo State University: Kuzbassvuzizdat. https://publications.hse.ru/books/71885100; (In Russian)

Distance Employment in the World. https://www.google.com/search?q=distant + Employment in the world \& tbm=isch\&ved=2ahUKEwiz6sjj5_ToAhWKOoUKHTq5C3cQ2-cCegQIABAA\&oq; (In Russian)

Konobevtsev, F., (2011). Remote work as a new form of employment // Kadrovik. Personnel management. (9):47-7. http:// трудовые-договоры.рф/article/385; (In Russian)

Kotlyarov, I. D., (2015). Non-standard forms of employment: positive, negative, neutral // Journal of Economic Regulation. 6. 4. 3. https://cyberleninka.ru/article/n/ (In Russian);

What is distance employment in Russia? http://www.iksmedia.ru/news/5254023-Kakova-distancionnaya-zanyatost-v. html\#ixzz6JnTIH9d7; (In Russian)

Musaev, B.A., (2017). Non-standard employment as a form of realizing the conditions of the new economic order. Science, 9, 2. http://naukovedenie.ru/PDF/59EVN217.pdf. https://cyberleninka.ru/article/n/; (In Russian)

Musaev, B.A., (2019). The growth of distance employment as a new trend in the development of the Russian labor market. Social and Labor Research, 2 (35). https://www.vcot.info/assets/img/magazine/issues/2019/35.pdf; (In Russian)

Moskaleva, M.V., (2019). Distance employment in the world. Legal distance employment: benefits for the state, company Workle - Moscow State University of Economics, Statistics and Informatics. https://www.slideshare.net/OKorotkova/16052014workle; (In Russian)

Martynova, O. V., (2016). Remote employment in Russia and the prospects for its development / OV Martynova. - Text: direct, electronic // Young scientist .. 2 (106). URL: https://moluch.ru/archive/106/25337/; https://yandex.ru/search/?text; (In Russian)

Razumov, A., Tsygankova, I., Antonova, G., \& Nikishina O. (2019). Non-Standard work as a form of using working time (Russian and foreign experience). Moscow. Publishing house - Dashkov and K. (In Russian) 